\title{
ARTICLE
}

Cancer Metabolism

\section{Activation of the reverse transsulfuration pathway through NRF2/CBS confers erastin-induced ferroptosis resistance}

\author{
Nan Liu ${ }^{1}$, Xiaoli Lin ${ }^{1}$ and Chengying Huang ${ }^{1}$
}

\begin{abstract}
BACKGROUND: Ferroptosis is an iron-dependent, lipid peroxide-mediated cell death that may be exploited to selective elimination of damaged and malignant cells. Recent studies have identified that small-molecule erastin specifically inhibits transmembrane cystine-glutamate antiporter system $\mathrm{x}_{\mathrm{c}}{ }^{-}$, prevents extracellular cystine import and ultimately causes ferroptosis in certain cancer cells. In this study, we aimed to investigate the molecular mechanism underlying erastin-induced ferroptosis resistance in ovarian cancer cells.

METHODS: We treated ovarian cancer cells with erastin and examined cell viability, cellular ROS and metabolites of the transsulfuration pathway. We also depleted cystathionine $\beta$-synthase (CBS) and NRF2 to investigate the CBS and NRF2 dependency in erastin-resistant cells.

RESULTS: We found that prolonged erastin treatment induced ferroptosis resistance. Upon exposure to erastin, cells gradually adapted to cystine deprivation via sustained activation of the reverse transsulfuration pathway, allowing the cells to bypass erastin insult. CBS, the biosynthetic enzyme for cysteine, was constantly upregulated and was critical for the resistance. Knockdown of CBS by RNAi in erastin-resistant cells caused ferroptotic cell death, while CBS overexpression conferred ferroptosis resistance. We determined that the antioxidant transcriptional factor, NRF2 was constitutively activated in erastin-resistant cells and NRF2 transcriptionally upregulated CBS. Genetically repression of NRF2 enhanced ferroptosis susceptibility.

CONCLUSIONS: Based on these results, we concluded that constitutive activation of NRF2/CBS signalling confers erastin-induced ferroptosis resistance. This study demonstrates a new mechanism underlying ferroptosis resistance, and has implications for the therapeutic response to erastin-induced ferroptosis.
\end{abstract}

British Journal of Cancer (2020) 122:279-292; https://doi.org/10.1038/s41416-019-0660-x

\section{BACKGROUND}

System $\mathrm{x}_{\mathrm{c}}{ }^{-}$is a transmembrane cystine-glutamate antiporter that specifically imports extracellular L-cystine into cells in exchange for glutamate. ${ }^{1,2}$ Cystine is an oxidised, disulfide form of cysteine. Due to intracellular reducing context, cystine imported into the cell by system $\mathrm{x}_{\mathrm{c}}{ }^{-}$is reduced to cysteine, which is required for the synthesis of glutathione (GSH), the key cellular antioxidant component, maintaining intracellular redox homoeostasis. ${ }^{3,4}$ Many cells rely on system $\mathrm{x}_{\mathrm{c}}{ }^{-}$to take up cystine, but this uptake is the rate-limiting step in obtaining cysteine. Blocking or inhibiting this process causes cystine deprivation, and perturbs cellular redox state. $^{5}$

System $\mathrm{x}_{\mathrm{c}}{ }^{-}$is a heterodimer composed of two distinct proteins: the substrate-specific subunit xCT also known as SLC7A11 (solute carrier family 7), which is responsible for the transporter function of system $\mathrm{x}_{\mathrm{c}}{ }^{-}$, and heavy chain of $4 \mathrm{~F} 2$ cell surface antigen (4F2hc) SLC3A2 (solute carrier family 3 member 2). ${ }^{6,7}$ Recent studies have identified that small-molecule erastin specifically inhibits $x C T$, resulting in depletion of cellular GSH, and iron-dependent cell death, designated as ferroptosis. ${ }^{8}$ Ferroptosis is featured by irresolvable lipid peroxidation, which is mechanistically triggered by ferrous iron $\left(\mathrm{Fe}^{2+}\right)$-mediated Fenton reaction. ${ }^{9-12}$ Deprivation of cysteine by system $\mathrm{x}_{\mathrm{c}}{ }^{-}$blockage leads to ferroptosis through
GSH depletion and lipid peroxidation in some cell contexts, while some types of cells are tolerant to system $\mathrm{x}_{\mathrm{c}}{ }^{-}$inhibition.

In addition to importing cystine by system $\mathrm{x}_{\mathrm{c}}{ }^{-}$, mammalian cells can obtain cysteine through the reverse transsulfuration pathway, which is the only route for cysteine biosynthesis. ${ }^{13-15}$ The pathway channels dietary-derived methionine to S-adenosyl homocysteine and homocysteine. Cystathionine $\beta$-synthase (CBS) catalyses the condensation of homocysteine with serine to generate cystathionine, which cystathione $\gamma$-lyase (CSE) converts to cysteine. ${ }^{16}$ Disruption of the transsulfuration pathway is associated with pathological conditions of several diseases such as vascular dysfunction ${ }^{17}$ and Huntington's disease. ${ }^{18,19}$ Previous studies have reported that certain types of cancer cells, which rely on system $\mathrm{x}_{\mathrm{c}}{ }^{-}$for cystine uptake due to their inability to synthesise cysteine via the transsulfuration pathway, are particularly sensitive to erastin insult. ${ }^{20-22}$ Therefore, understanding the molecular mechanisms underlying the crosstalk between ferroptosis and the transsulfuration pathway will contribute to the design of therapeutic approaches to regulate cancer cell fate.

In this study, we observed erastin-induced ferroptosis resistance in ovarian cancer cells. We found that the transsulfuration pathway was elevated due to CBS upregulation. Enhanced flux through the transsulfuration pathway provided cells with cysteine,

${ }^{1}$ Division of Obstetrics and Gynecology, Nanfang Hospital, Southern Medical University, Guangzhou 510515, China

Correspondence: Nan Liu (liunan@smu.edu.cn)

Received: 11 June 2019 Revised: 2 November 2019 Accepted: 8 November 2019

Published online: 10 December 2019 
which compensated the shortage of cysteine by system $\mathrm{x}_{\mathrm{c}}{ }^{-}$ blockage. We demonstrated that constitutive activation of NRF2/ CBS signalling conferred erastin-induced ferroptosis resistance.

\section{METHODS}

Cell culture and reagents

Human ovarian cancer SKOV3 and OVCA429 cells were maintained in RPMI 1640 (SKOV3 cells) or DMEM (OVCA429 cells) medium supplemented with $10 \%$ foetal bovine serum and $1 \%$ penicillin/ streptomycin at $37^{\circ} \mathrm{C}$ with $5 \% \mathrm{CO}_{2}$ in a humidified atmosphere. To establish erastin-resistant cells, SKOV3 cells were treated with $10 \mu \mathrm{M}$ erastin for 7 days, OVCA429 cells were treated with $20 \mu \mathrm{M}$ erastin for 5 days and the remaining cells were washed and cultured in fresh medium. Cell viability was analysed by using the Cell Counting Kit-8 (CCK-8) (Sigma \#96992) according to the manufacturer's instructions. Liproxstatin-1 (Lipo-1), 7-Cl-O-Nec1 (Nec-1s) and Z-VAD-FMK were obtained from Selleckchem; erastin, ferrostatin-1 (Fer-1), N-acetyl cysteine (NAC), desferrioxamine (DFO), Trolox, necrosulfonamide (NSA), sulfasalazine (SAS) RSL3 and FIN56 were purchased from Sigma. $\mathrm{CM}-\mathrm{H}_{2}$ DCFDA and $\mathrm{C11-}$ BODIPY (581/591) were purchased from Molecular Probes, Invitrogen.

ROS analysis

Cells were incubated with $5 \mu \mathrm{M} \mathrm{CM}-\mathrm{H}_{2}$ DCFDA for ROS, or $5 \mu \mathrm{M}$ C11-BODIPY (581/591) for lipid ROS in the dark for $30 \mathrm{~min}$ at $37^{\circ} \mathrm{C}$. Cells were then harvested and resuspended in phenol-red-free medium. Fluorescence was analysed on BD FACsCalibur system with CellQuest Pro software.

\section{Lipid peroxidation assay}

Malondialdehyde (MDA), the end product of lipid peroxidation, was assessed by using a lipid peroxidation (MDA) assay kit (\#MAK085, Sigma). Cells were homogenised on ice in MDA lysis buffer, followed by centrifugation at $13,000 \times g$ for 3 min. MDA in cell lysate reacts with thiobarbituric acid (TBA) solution to generate MDA-TBA adduct, which is colorimetric $(O D=532 \mathrm{~nm})$ and is proportional to the MDA present.

Glutathione (GSH) assay

Cells were collected and homogenised in a 5\% 5-sulfosalicylic acid (SSA) solution. The reduced GSH concentration in cell lysates was measured by using the Reduced Glutathione (GSH) Assay kit (\# K464-100, BioVision) as per the manufacturer's instructions. The results were normalised to total protein concentration for each sample.

\section{Glutamate release assay}

The release of glutamate from cells into the extracellular medium was assayed as described. ${ }^{23}$ Cells were washed with cystine uptake buffer $(137 \mathrm{mM}$ choline chloride, $3 \mathrm{mM} \mathrm{KCl}, 1 \mathrm{mM} \mathrm{CaCl}, 1 \mathrm{mM}$ $\mathrm{MgCl}_{2}, 5 \mathrm{mM}$ D-glucose, $0.7 \mathrm{mM} \mathrm{K}_{2} \mathrm{HPO}_{4}, 10 \mathrm{mM}$ HEPES and $300 \mu \mathrm{M}$ cystine, $\mathrm{pH}$ 7.4), then incubated with uptake buffer containing DMSO or $10 \mu \mathrm{M}$ erastin for $60 \mathrm{~min}$ at $37^{\circ} \mathrm{C}$. Glutamate in cell medium was detected by using Amplex Red Glutamic Acid/Glutamate Oxidase Assay kit (\# A-12221, Thermo Fisher) as per the manufacturer's instructions. Cell number was quantified by trypan blue exclusion assay. Glutamate release was normalised to cell number.

Cystine uptake assay

Cells were washed three times with pre-warmed sodium-free uptake buffer $(137 \mathrm{mM}$ choline chloride, $3 \mathrm{mM} \mathrm{KCl}, 1 \mathrm{mM} \mathrm{CaCl}$, $1 \mathrm{mM} \mathrm{MgCl} 2,5 \mathrm{mM}$ D-glucose, $0.7 \mathrm{mM} \mathrm{K}_{2} \mathrm{HPO}_{4}$ and $10 \mathrm{mM}$ HEPES, $\mathrm{pH} 7.4$ ), then incubated with $0.5 \mathrm{ml}$ of pre-warmed uptake buffer at $37^{\circ} \mathrm{C}$ for $10 \mathrm{~min}$. The buffer was then replaced with $0.5 \mathrm{ml}$ of $\left[{ }^{14} \mathrm{C}\right]$ cystine $(0.2 \mu \mathrm{Ci} / \mathrm{mL})$ uptake buffer and incubated at $37^{\circ} \mathrm{C}$ for $3 \mathrm{~min}$. Cells were rapidly washed three times with ice-cold uptake buffer and lysed in $0.5 \mathrm{ml}$ of $0.1 \mathrm{M} \mathrm{NaOH}$. The radioactive counts per minute were assessed by a scintillation counter. ${ }^{24}$

\section{Metabolomic profiling}

Cell pellets $\left(\geq 2 \times 10^{6}\right.$ cells) were flash frozen in liquid nitrogen and stored at $-80^{\circ} \mathrm{C}$ until processing. Metabolites were profiled by Dian Diagnostics Co. Ltd. (Zhejiang, China). Samples were prepared by using aqueous methanol extraction process to remove the protein fraction while allowing maximum recovery of small molecules. For quality control (QC) purpose, a recovery standard was included before the extraction process. The extract was divided into four fractions: one for analysis by UPLC/MS/MS with positive ion mode electrospray ionisation (ESI) (positive mode), one for UPLC/MS/MS with negative ion mode ESI (negative mode), one for GC/MS and one for backup. Each sample was processed to remove the organic solvent, and prepared for either UPLC/MS/MS or GC/MS. For UPLC/MS/MS, the sample extracts were reconstituted in acidic or basic LC-compatible solvents. The acidic extracts were analysed by using positive mode, and gradient eluted by using water and methanol containing $0.1 \%$ formic acid, while the basic extracts were analysed by using negative mode and eluted by using water/methanol containing $6.5 \mathrm{mM}$ ammonium bicarbonate. The MS analysis alternated between MS and data-dependent $\mathrm{MS}^{2}$ scans using dynamic exclusion. For GC/MS analysis, samples were derived using bistrimethyl-silyl-triflouroacetamide (BSTFA), and analysed on a Thermo-Finnigan Trace DSQ fast-scanning single-quadrupole mass spectrometer using electron impact ionisation. Raw data were extracted and peak-identified using Metabolon's hardware and software. Compounds were identified by comparison with library entries of purified standards on both the LC and GC platforms. Each biochemical value was normalised to cell number and scaled to its median value. The unpaired $t$ test for differential abundance with a standard two-tailed and two-sample $t$ test was performed using mattes function in MATLAB on each metabolite to calculate the Wilcoxon $t$ statistic for two groups being compared. The resultant $p$-value of less than 0.05 was selected as significant. The experiment was conducted in biological quintuplicate.

\section{Western blotting}

Cell pellets were lysed in RIPA buffer $(25 \mathrm{mM}$ Tris- $\mathrm{HCl}, \mathrm{pH}=7.5$, $150 \mathrm{mM} \mathrm{NaCl}, 0.1 \%$ Nonidet P-40, 0.5\% sodium deoxycholate and $0.1 \%$ SDS) supplemented with protease inhibitor cocktail (Sigma) and phosphatase Inhibitor Cocktail 2 and 3 (Sigma). The lysates were cleared and subjected to SDS-PAGE and transferred onto nitrocellulose membranes. The following antibodies were used: anti-CBS (\#14782, Cell Signaling Technology [CST]), anti-CSE (\#30068, CST), anti-NRF2 (\#12721, CST), and anti-GAPDH (sc32233, Santa Cruz Biotechnology).

siRNA reverse transfection and lentivirus infection About $10 \mathrm{nM}$ of siRNA in OptiMEM $(250 \mu \mathrm{l})$ was mixed with $250 \mu \mathrm{l}$ of OptiMEM with $2.5 \mu \mathrm{l}$ of Lipofectamine RNAiMAX (Invitrogen) for $20 \mathrm{~min}$, then with $1.5 \mathrm{ml}$ of cell suspension in regular medium. A total of 200,000 cells per well were seeded to sixwell plates. Cells were then analysed at indicated time points. All siRNAs were purchased from Sigma: siCtrl (SIC001), siCBS (SASI_Hs01_00214623) and siNRF2 (SASI_Hs01_00182393). Lentiviruses carrying CBS or empty vector were previously described. ${ }^{25}$ HEK293T cells were transfected by $\mathrm{pCDH}-\mathrm{hCBS}$ or $\mathrm{pCDH}$ vector constructs along with packaging constructs as per Lipofectamine 2000 manual.

qRT-PCR

RNA was extracted using Trizol reagent (Invitrogen). cDNA was synthesised using SuperScript ${ }^{\mathrm{TM}}$ III reverse transcriptase and oligo-dT (Life Technologies). qPCR was performed using the iTaq universal SYBR green supermix (Bio-rad). GAPDH was used for normalisation 


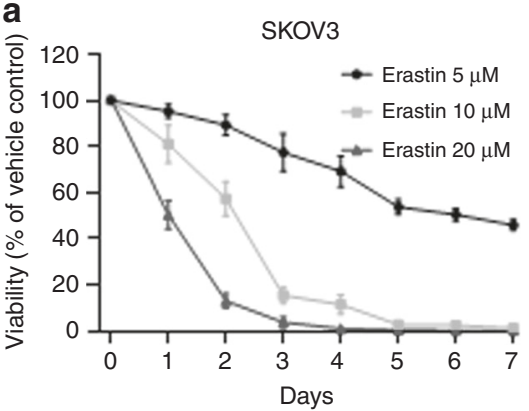

b

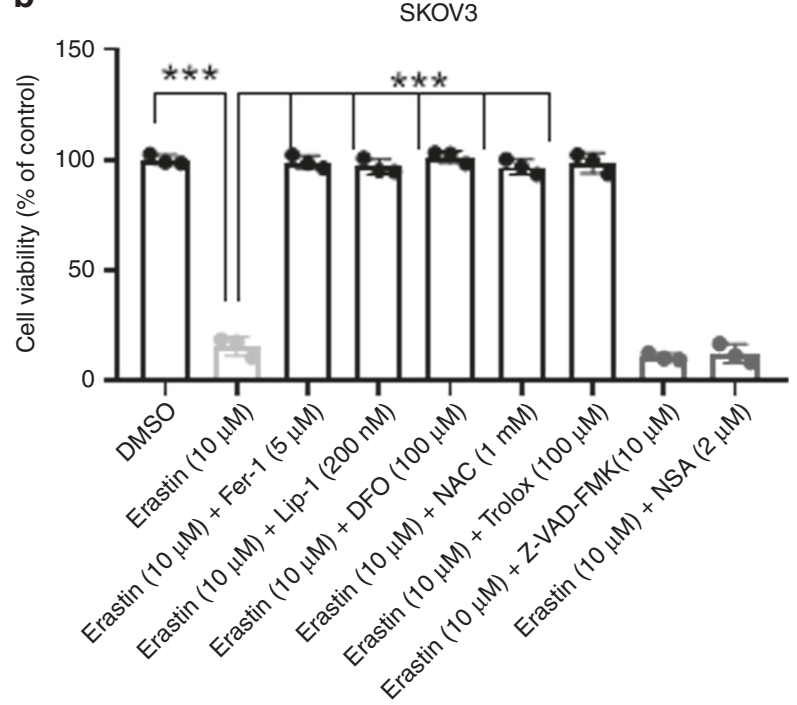

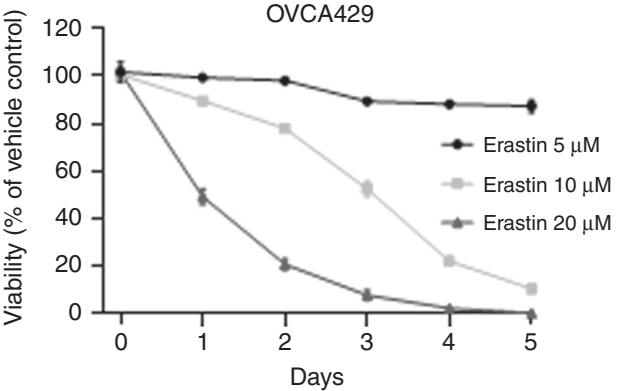

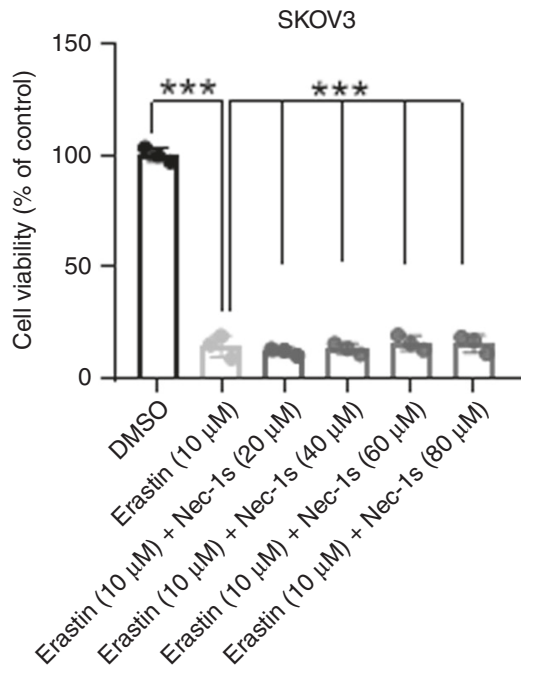

OVCA429

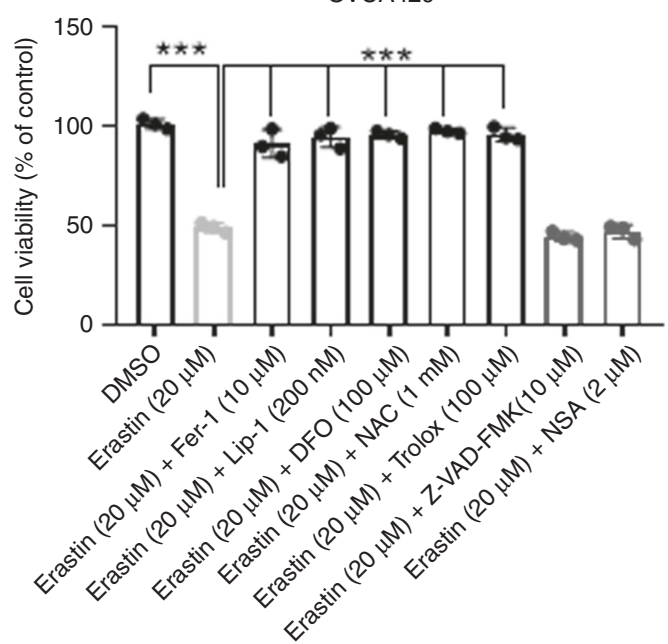

OVCA429

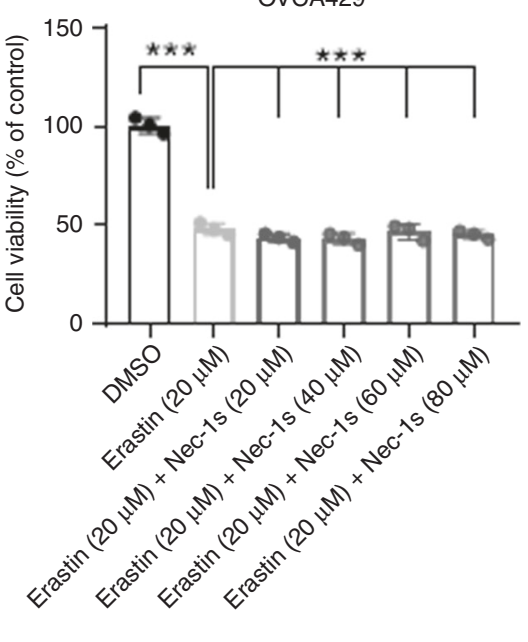

Fig. 1 Lethal dose of erastin induces ferroptosis resistance in ovarian cancer cells. a SKOV3, OVCA429 cells were sensitive to erastin-induced death. SKOV3, OVCA429 cells were treated with erastin $(0,5,10$ and $20 \mu \mathrm{M})$ for the indicated time points, and cell viability was analysed. b Fer1, Lip-1, DFO, NAC and Trolox rescued erastin-induced death, confirming erastin-induced ferroptosis in SKOV3 and OVCA429 cells. Cells were treated with erastin in the absence or presence of indicated inhibitors (Fer-1, Lipo-1, BSO, NAC, Troxl, z-VAD-FMK, NAS and Nec-1s) for $24 \mathrm{~h}$, and cell viability was assayed, ${ }^{* * *} p<0.0001$. c Outline of the establishment of SKOV3 and OVCA429 erastin-resistant cells. d SKOV3 Era-R cells were resistant to lethal dosage of erastin. SKOV3 and SKOV3 Era-R cells were treated with erastin $20 \mu \mathrm{M}$ for $24 \mathrm{~h}$. Cell viability was monitored by light microscopy (scale bar $=400 \mu \mathrm{m}$, magnification: $\times 10$ ). e SKOV3 Era-R, OVCA429 Era-R cells were resistant to erastin insult. Cells were treated with erastin $(0,10,20$ and $40 \mu \mathrm{M})$ for $72 \mathrm{~h}$, and cell viability was examined, $* * * p<0.0001$. f SKOV3, SKOV3 Era-R, OVCA429 and OVCA429 Era-R cells were either untreated or treated with SAS $(1 \mathrm{mM})$, RSL $(1 \mu \mathrm{M})$ or FIN56 $(5 \mu \mathrm{M})$ for the indicated time, cell viability was examined. ${ }^{* *} p<0.001,{ }^{* * *} p<0.0001$ as compared with untreated control. 

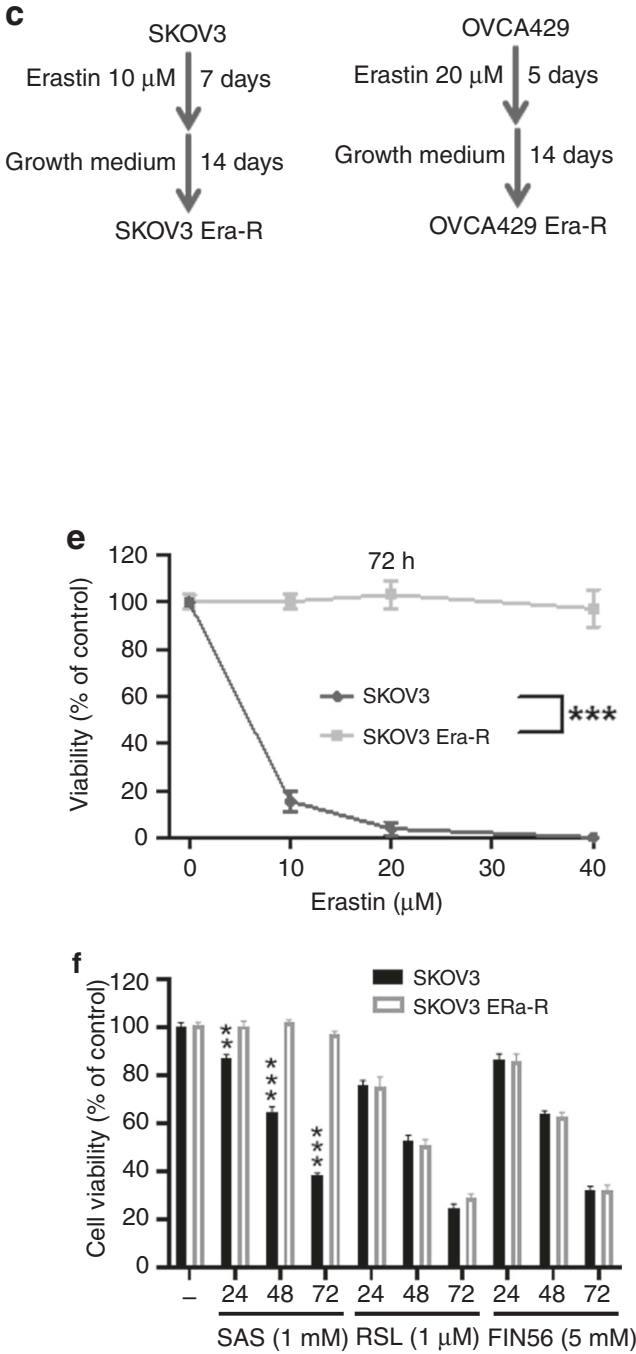

Fig. 1 Continued

in all $\mathrm{qPCR}$ assays. Fold changes were analysed by the $2-\Delta \Delta C T$ method for relative quantification. The primers used for qPCR were as follows: CBS: 5'-GGCCAAGTGTGAGTTCTTCAA-3' and 5'-GGCTC GATAATCGTGTCCCC-3'; CSE: 5'-CATGAGTTGGTGAAGCGTCAG-3' and 5'-AGCTCTCGGCCAGAGTAAATA-3'; GAPDH: 5'-CATGGGTGT GAACCATGAGA-3' and 5'-CAGTGATGGCATGGACTG-TG-3'.

Measurement of hydrogen sulfide $\left(\mathrm{H}_{2} \mathrm{~S}\right)$

$\mathrm{H}_{2} \mathrm{~S}$ was determined in erastin-resistant cells following the protocol as previously described. ${ }^{26,27}$ Cells were lysed in a lysis buffer (potassium phosphate buffer $100 \mathrm{mM}$, pH 7.4, sodium orthovanadate $10 \mathrm{mM}$ and proteases inhibitors). Protein concentration was determined by Pierce BCA Protein Assay Kit. Cell lysates were incubated with a reaction mixture containing pyridoxal-5-phosphate $(2 \mathrm{mM})$ at $37^{\circ} \mathrm{C}$ for $30 \mathrm{~min}$. Next, trichloroacetic acid solution (10\%) was added to each sample followed by zinc acetate (1\%). Then, N,N-dimethyl-p-phenylendiamine sulfate (DPD, $20 \mathrm{mM})$ in $\mathrm{HCl}(7.2 \mathrm{M})$ and $\mathrm{FeCl}_{3}(30 \mathrm{mM})$ in $\mathrm{HCl}(1.2 \mathrm{M})$ were added, and the absorbance of the solutions was measured at a wavelength of $650 \mathrm{~nm}$. Aminooxyacetic acid (AOAA, $1 \mathrm{mM}$ ), a CBS inhibitor, was added to the reaction mixture in some samples, which served as a positive control of CBS inhibition. $\mathrm{H}_{2} \mathrm{~S}$ production was plotted against a calibration curve of $\mathrm{NaHS}$ $(3-250 \mu \mathrm{M})$. Data were presented as nanomoles per milligram of total protein per minute.
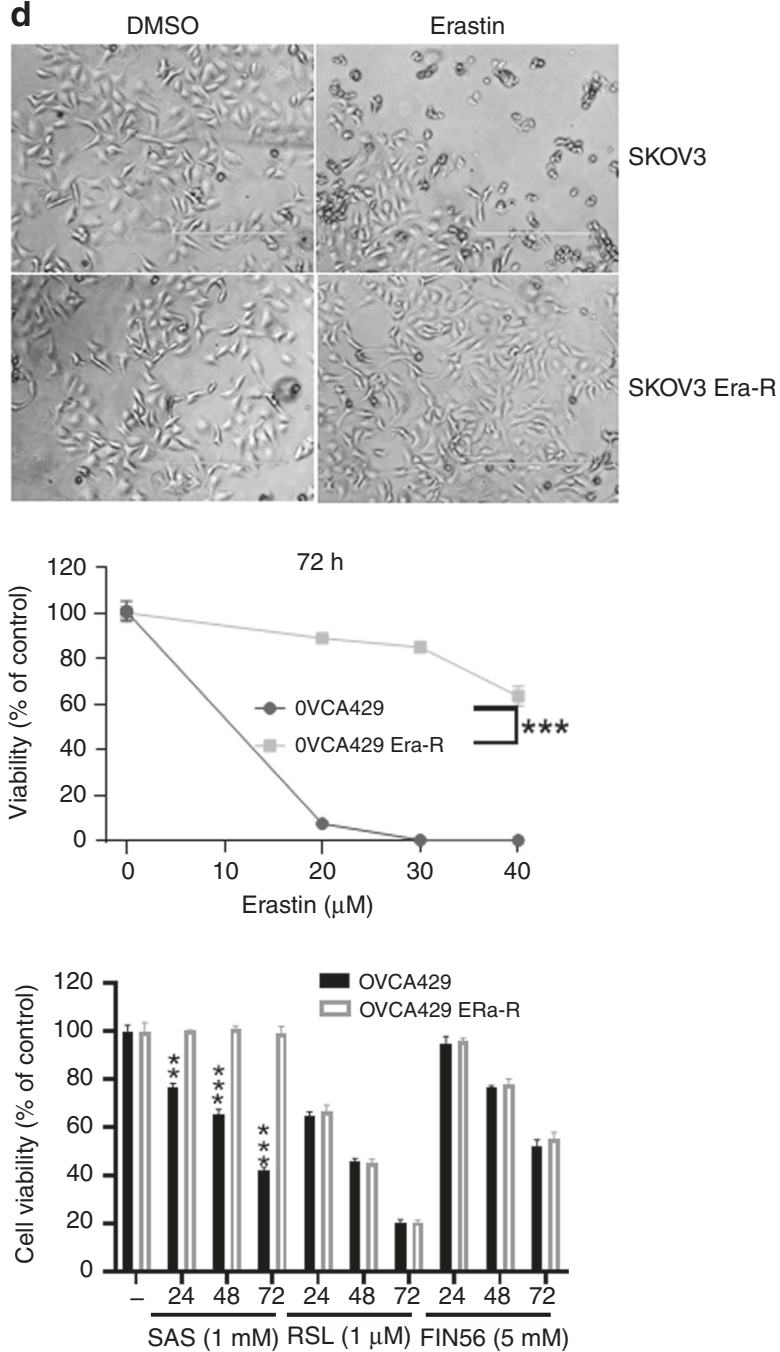

Plasmid constructions and reporter activity assay The 1200-bp DNA fragment containing the CBS promoter region from -1000 to $+200 \mathrm{bp}$ was amplified from SKOV3 Era-R cell genomic DNA by PCR with the following primers: CBS-Kpnl forward, 5'-GGTACCGACATTTAATTCTAATTCACGTCTC-3' and CBSBg/ll reverse, 5'-AGATCTGTCCAGAGAGGGGAGCGAGTCTCGG-3'. This fragment was cloned into the pGL3-basic luciferase reporter vector (Promega), using $\mathrm{Kpnl}$ and $\mathrm{Bg} / \mathrm{ll}$ restriction sites. This construct containing the human CBS promoter was named pGL3phCBS. The NRF2-binding antioxidant response element (ARE) deletion mutant of CBS promoter-luciferase construct pGL3phCBS- $\triangle \mathrm{ARE}$ was generated by the overlapping PCR technique and ligated into pGL3-basic luciferase reporter vector (Promega). The following two primer sets were used: (I) CBS-Kpnl forward primer and reverse primer, 5'-CGGCGACCCCGGGGTGGGGACC CACGGCGA-3' and (II) forward primer, 5'-TCGCCGTGGGTCCCC ACCCCGGGGTCGCCG-3' and CBS-Bglll reverse primer. The pCDNA3-Myc3-NRF2 was a gift from Yue Xiong (Addgene plasmid \#21555). The pCDNA3 empty vector was made from pCDNA3Myc3-NRF2 by removing Myc3-NRF2 sequence and religating the vector. A pCH110 plasmid encoding $\beta$-galactosidase ( $\beta$-gal) was purchased from Amersham. To determine the effect of NRF2 on the CBS promoter activity, SKOV3 Era-R cells were seeded in 24well plates and transiently cotransfected with each reporter plasmid, pCDNA3-Myc3-NRF2 or pCDNA3 empty vector, and 
a
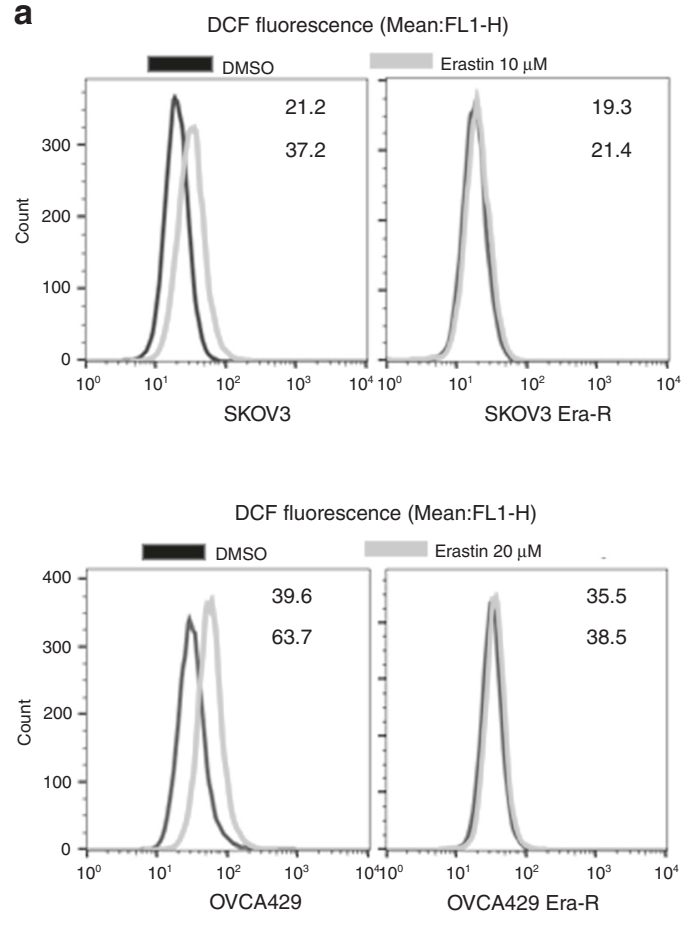

b

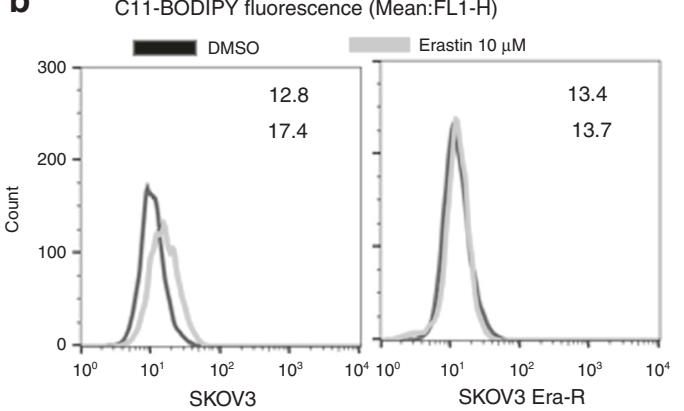

C11-BODIPY fluorescence (Mean:FL1-H)



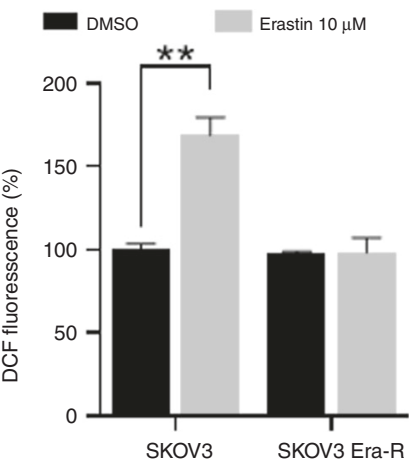
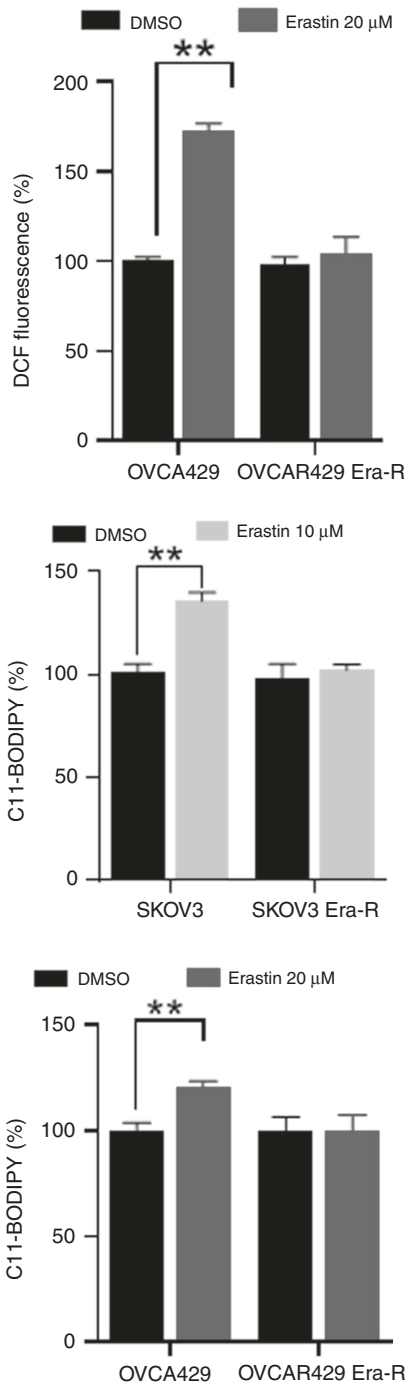

Fig. 2 Adaption to erastin insult reprograms cellular cysteine metabolism. a-d Cells were treated with erastin or DMSO for $8 \mathrm{~h}$. Cellular and lipid ROS were assayed by flow cytometry using $\mathrm{H}_{2}$ DCFDA (a) and C11-BODIPY (b), respectively. The lipid peroxidation marker, MDA (c), and GSH content (d) were measured, ${ }^{* *} p<0.001$. e Cells were treated with DMSO or erastin for $8 \mathrm{~h}$. Glutamate release from cells to culture medium was examined, ${ }^{* *} p<0.001$. f Cells were exposed to erastin for $8 \mathrm{~h}$. The activity of cystine uptake was measured, ${ }^{* * *} p<0.001$. g Analysis of metabolites of the transsulfuration pathway. Intracellular S-adenosyl homocysteine, homocysteine, cystathionine and cysteine are shown.

pCH110 plasmid using Lipofectamine 2000 reagent (Invitrogen). After incubation for $24 \mathrm{~h}$, transfected cells were subjected to a luciferase activity assay using Luciferase Assay System (Promega). Relative luciferase activity was normalised to $\beta$-gal activity as described previously.
Statistical analysis

All of the results are mean values $\pm S D$ of three biological replicates. One-way ANOVA followed by Dunnett's multiple comparisons test was performed using GraphPad Prism version 8.0.0 for Windows (GraphPad Software, San Diego, California USA, www.graphpad.com). 

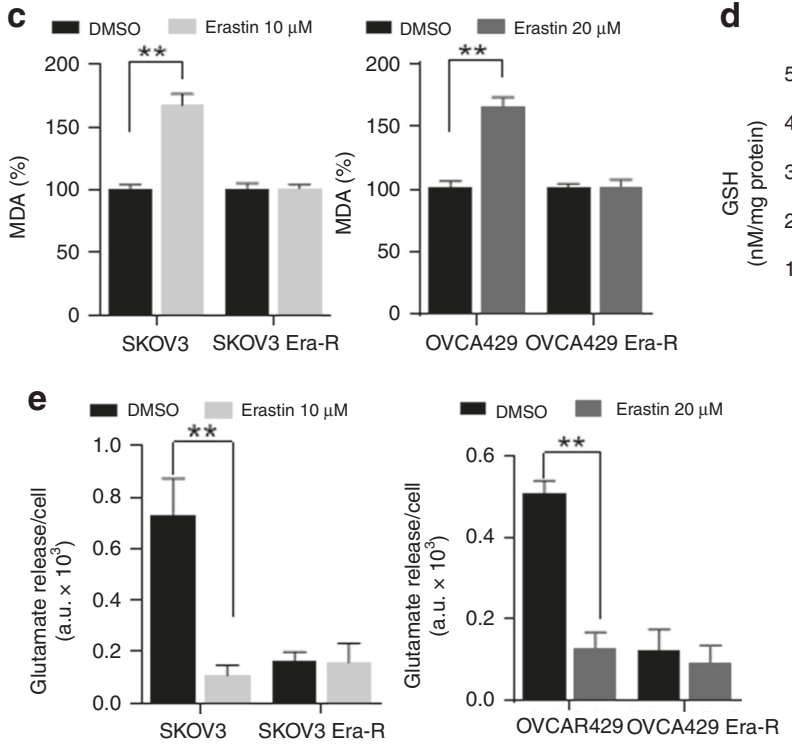

g
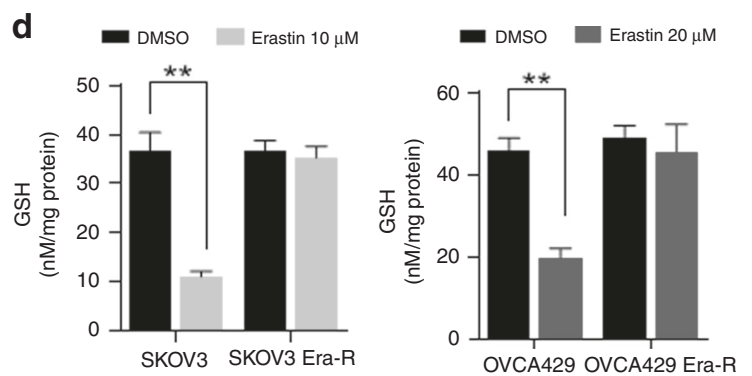

f
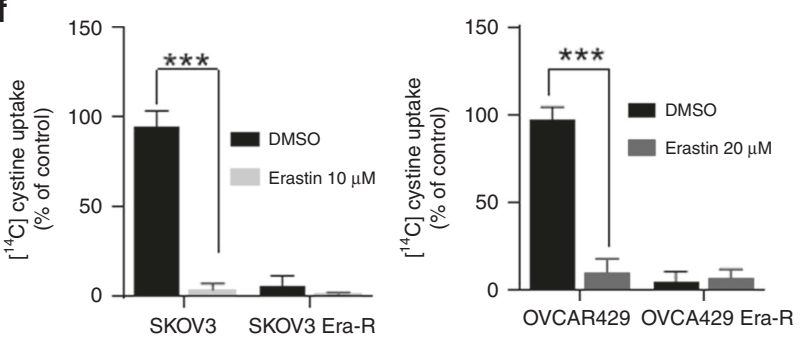
S-adenosyl homocysteine
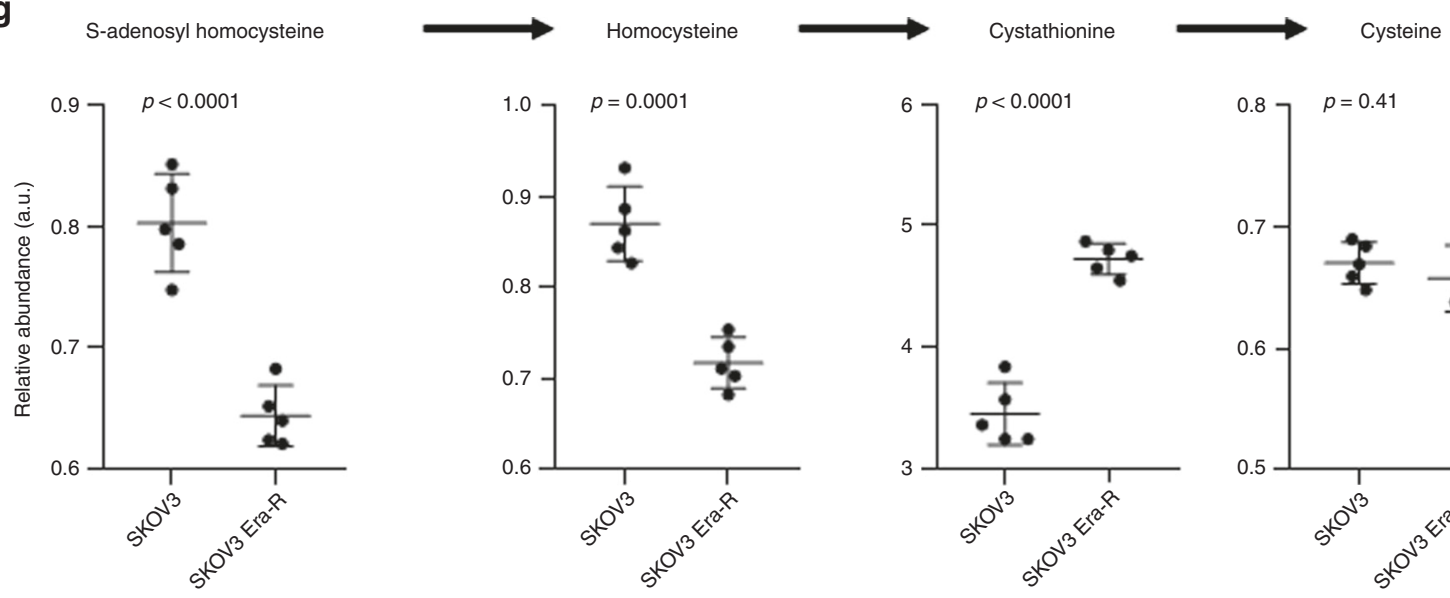

S-adenosyl homocysteine
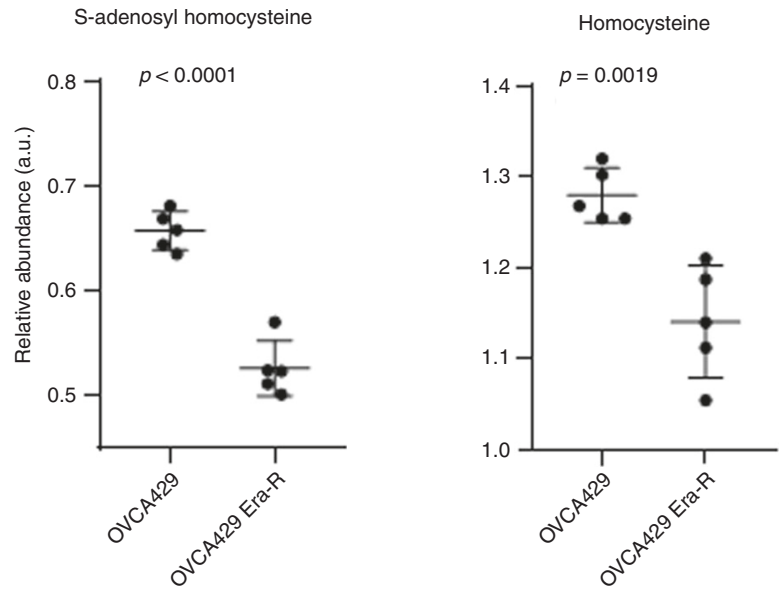

Fig. 2 Continued

\section{RESULTS}

Lethal dose of erastin induces ferroptosis resistance in ovarian cancer cells

We initially set out to investigate the ferroptosis susceptibility of ovarian cancer cells in response to erastin. SKOV3 and OVCA429 cells were sensitive to erastin-induced cell death (Fig. 1a). The ferroptosis inhibitors ferrostatin-1 (Fer-1), liproxstatin-1 (Lip-1), iron chelator deferoxamine (DFO) and the antioxidants NAC and

Trolox $^{8,28}$ rescued the lethal erastin, while the apoptosis inhibitor Z-VAD-FMK, ${ }^{29}$ and the necroptosis inhibitors necrosulfonamide (NSA), 7-Cl-O-Nec1 (Nec-1s) ${ }^{28}$ failed to block erastin-induced death (Fig. 1b), indicating that erastin is a potent ferroptosis inducer in the tested cell lines. We noticed that some cells still survived even when treated with lethal dose of erastin for 5-7 days. These cells were recovered in fresh growth medium and displayed significant resistance to the erastin insult, therefore, they were named as 


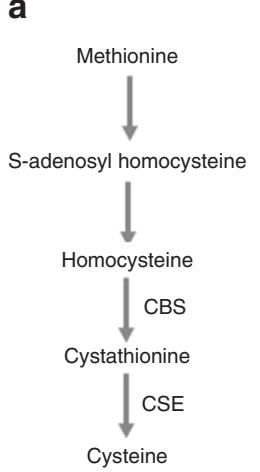

b

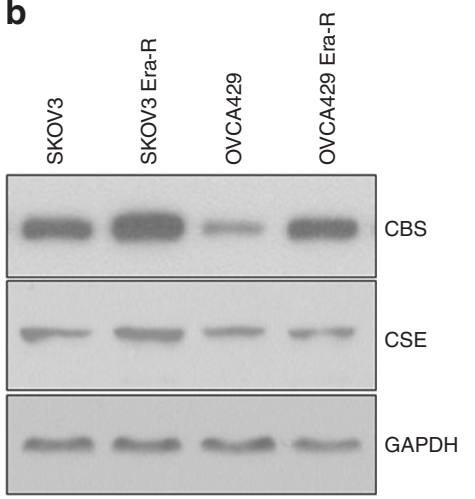

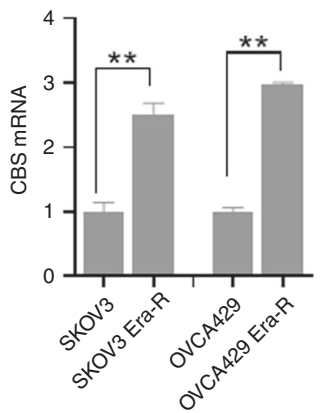

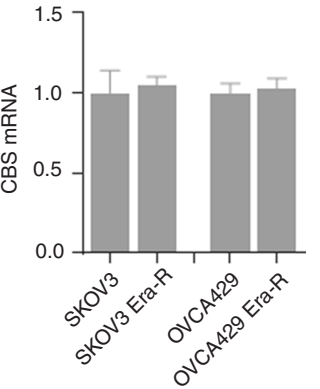

d

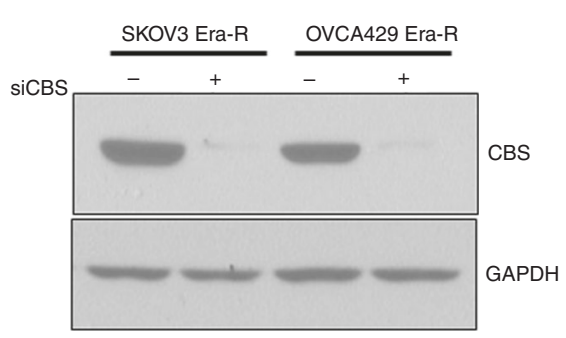

e
SKOV3 Era-R OVCA429 Era-R $\begin{array}{ccc}\text { SKOV3 Era-R } & \text { OVCA429 Era-R } \\ \mathbf{n} \text { siCtrl } & \text { sicBS } & \text { siCtrl }\end{array}$

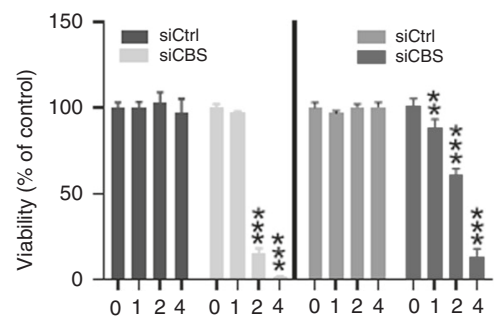

Days post siRNA transfection

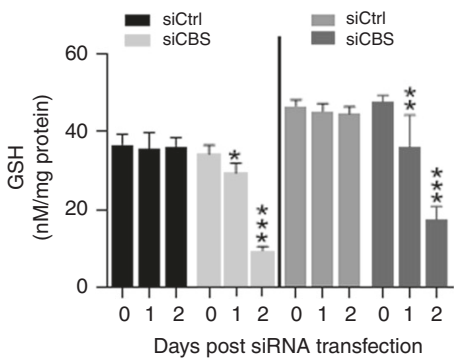

g

DCF fluorescence (Mean:FL1-H)
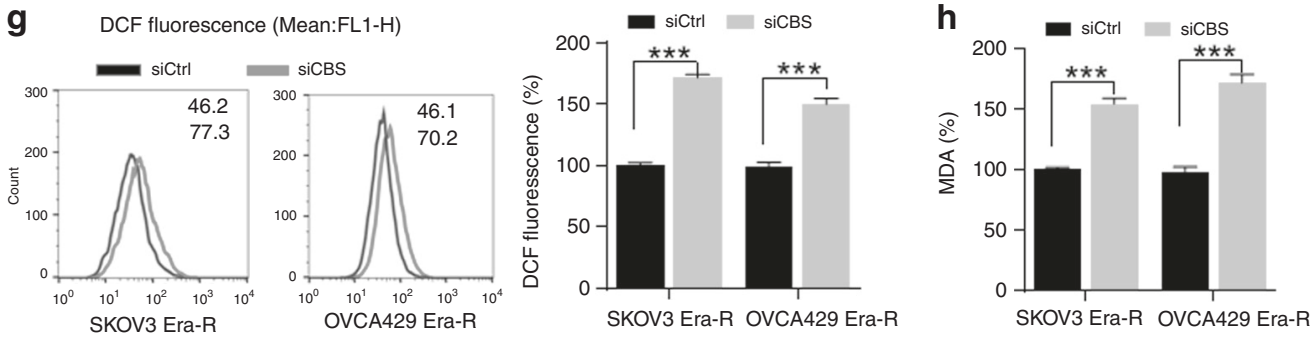

C11-BODIPY fluorescence (Mean:FL1-H)
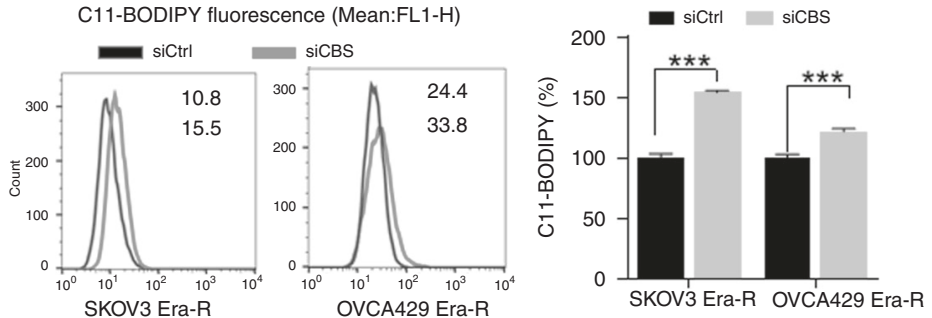

Fig. 3 Constitutive upregulation of CBS confers ferroptosis resistance. a An overview of the transsulfuration pathway channels methionine towards cysteine. $\mathbf{b}$ Cells were lysed and analysed for CBS and CSE expression by western blotting. c Comparison of CBS and CSE mRNA levels in erastin-resistant cells and their parental cells, ${ }^{* *} p<0.001$. d Cells were transfected with either scramble control siRNA or CBS siRNA for $48 \mathrm{~h}$. CBS knockdown was validated by western blotting. e Cells were transfected with either scramble control siRNA or CBS siRNA. Cell viability was examined over 4 days, ${ }^{* *} p<0.001,{ }^{* * *} p<0.0001$ as compared with the group at day 0 of transfection. $\mathbf{f}$ Cells were transfected with indicated siRNAs; GSH content was measured over $48 \mathrm{~h},{ }^{*} p<0.01,{ }^{* *} p<0.001,{ }^{* * *} p<0.0001$ as compared with the group at day 0 of transfection. $\mathbf{g}$, $\mathbf{h}$ Cells were transfected with indicated siRNAs for $48 \mathrm{~h}$; cellular ROS, lipid ROS (g) and MDA (h) were measured, ${ }^{* * *} p<0.0001$. $\mathbf{i}, \mathbf{j}$ Cells were transfected with siRNAs for $24 \mathrm{~h}$, followed by DMSO, Fer-1 or Lip-1 treatment for $24 \mathrm{~h}$. Lipid ROS (i) and cell viability (j) were measured, ${ }^{* * *} p<$ 0.0001 . k Basal level of hydrogen sulfide $\left(\mathrm{H}_{2} \mathrm{~S}\right)$ in cell homogenates was measured. The addition of CBS inhibitor (AOAA, $\left.1 \mathrm{mM}\right)$ served as a positive control for CBS inhibition. Data were presented as nanomoles per milligram of total protein per minute, ${ }^{* *} p<0.001$ as compared with the group with siCtrl and DMSO treatment.

SKOV3 Era-R and OVCA429 Era-R for erastin-resistant cells (Fig. 1c-e). The established erastin-resistant cell lines also displayed resistance to sulfasalazine (SAS), which like erastin, triggers ferroptosis through inhibition of system $X_{c}^{-8}$ (Fig. 1f). We tested the sensitivity of these erastin-resistant cell lines to the other types of ferroptosis inducers, RSL3 and FIN56, ${ }^{30}$ which induce ferroptosis by inhibiting the glutathione peroxidase GPX4, a downstream antioxidant enzyme of system $\mathrm{X}_{\mathrm{c}}{ }^{-}$. Over $72 \mathrm{~h}, \mathrm{RSL3}-$ and FIN56-induced growth inhibition in SKOV3 Era-R and
OVCA429 Era-R cells was on par with that in the parental SKOV3 and OVCA429 cells, respectively (Fig. 1f). This observation indicated that resistance was likely via modulation of upstream GPX4.

Adaption to erastin insult reprograms cellular cysteine metabolism Erastin inhibits the transmembrane cystine-glutamate antiporter system $\mathrm{X}_{\mathrm{c}}{ }^{-}$, blocks cystine influx, leading to intracellular cysteine shortage and consequently depletion of GSH, causing extensive 

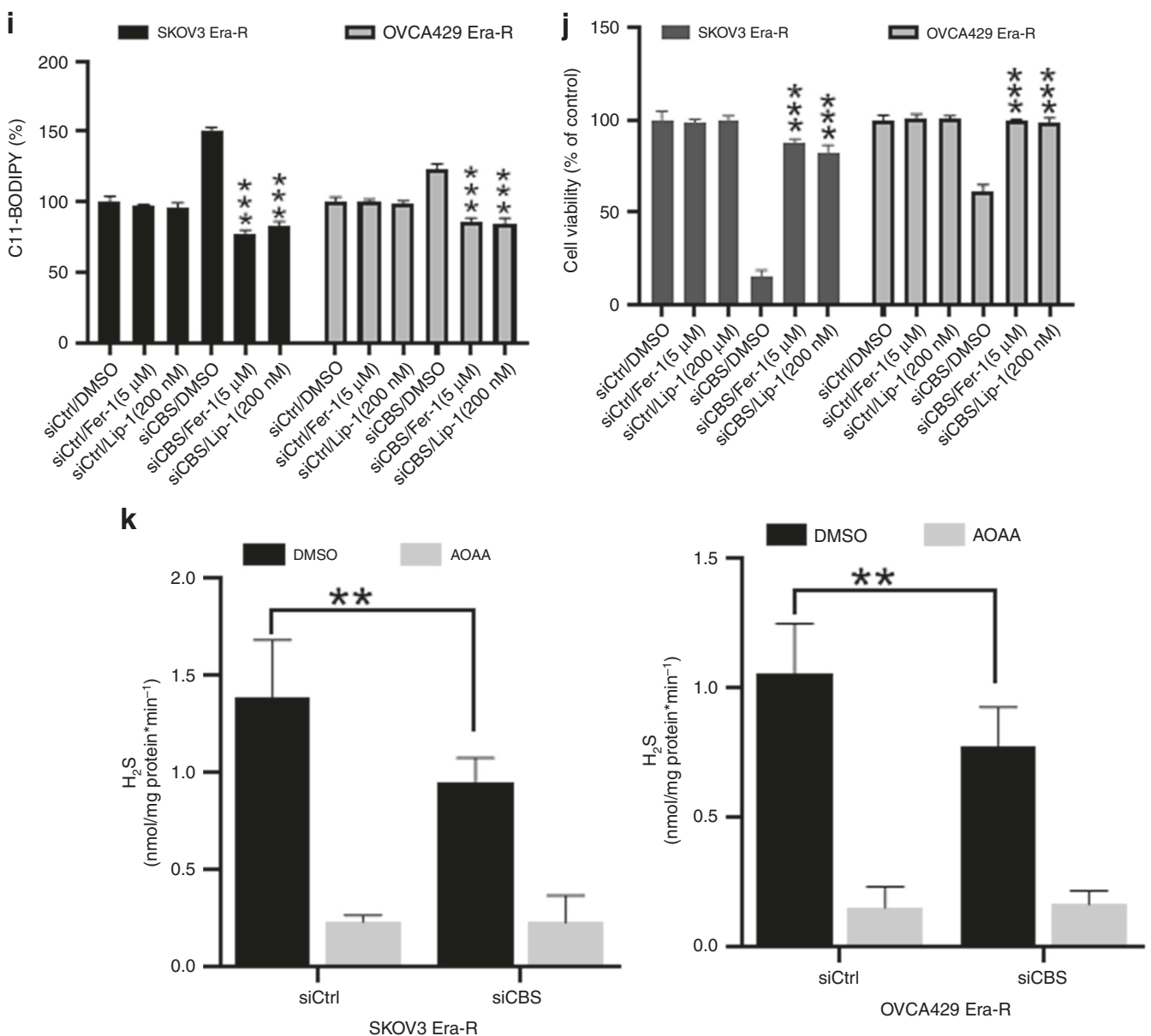

Fig. 3 Continued

lipid peroxidation, a featured event in ferroptosis. ${ }^{8}$ To gain insight into the erastin resistance, we treated cells with erastin and analysed cellular ROS and lipid peroxidation by flow cytometry using the fluorescent probes $\mathrm{H}_{2}$ DCFDA and C11-BODIPY, respectively. As expected, both SKOV3 and OVCA429 cells showed an increase in fluorescence after $8 \mathrm{~h}$ of treatment with erastin, whereas no increase in fluorescence was observed in SKOV3 Era-R and OVCA429 Era-R cells (Fig. 2a, b). We further examined the accumulation of the end product of lipid oxidation, malondialdehyde (MDA). ${ }^{31}$ As compared with vehicle-treated cells, erastin treatment caused much increase in MDA in SKOV3 and OVCA429 cells, but not in SKOV3 Era-R and OVCA429 Era-R cells (Fig. 2C). We also quantified GSH content in cells upon erastin treatment. Erastin-induced downregulation of GSH was clearly observed in SKOV3 and OVCA429 cells, while GSH content in SKOV3 Era-R and OVCA429 Era-R cells remained unaltered (Fig. $2 d$ ). These data together suggested that erastin-resistant cells adapted to erastin insult through a mechanism to rebuild up GSH homoeostasis. We monitored glutamate release to culture medium and confirmed that erastin acts via system $\mathrm{X}_{\mathrm{c}}{ }^{-}$inhibition (Fig. 2e); however, both SKOV3 Era-R and OVCA429 Era-R cells exhibited minimal glutamate release even without erastin treatment (Fig. 2e). We further compared the activity of system $\mathrm{X}_{\mathrm{c}}{ }^{-}$in parental cells and erastin-resistant cells by determining the uptake of $\left[{ }^{14} \mathrm{C}\right]$ cystine into cells. Erastin treatment markedly blocked the cystine uptake in parental cells, whereas cystine uptake was severely blocked in erastin-resistant cells even without erastin treatment (Fig. 2f). These data supported the previous observation that erastin irreversibly inhibits system $\mathrm{X}_{\mathrm{c}}{ }^{-32}$ and indicated the impaired system $X_{c}{ }^{-}$in erastin-resistant cells. Since system $X_{c}{ }^{-}$functions as a primary source of cysteine for GSH biosynthesis, these erastinresistant cells might have developed an alternative source to provide cysteine, compensating the shortage of cystine influx resulting from system $\mathrm{X}_{\mathrm{c}}^{-}$blockage. We wondered whether erastin-resistant cells rely on the activation of transsulfuration pathway to synthesise cysteine. To this end, we performed global metabolic profiling on cellular extracts from SKOV3, SKOV3 Era-R, OVCA429 and OVCA429 Era-R cells and analysed metabolites in the transsulfuration pathway. There was no change in the level of cysteine in both parental and erastin-resistant cells. We observed an elevated flux through the transsulfuration pathway upstream of cysteine synthesis in erastin-resistant cells. The levels of $\mathrm{S}$ adenosyl homocysteine and homocysteine were decreased in both SKOV3 Era-R and OVCA429 Era-R cells as compared with their parental cells, but a significant increase in cystathionine levels was detected in erastin-resistant cells (Fig. 2g). These data suggested that the upregulated transsulfuration pathway for cysteine synthesis in erastin-resistant cells compensated for cystine deprivation by system $\mathrm{X}_{\mathrm{c}}{ }^{-}$blockage.

Constitutive upregulation of CBS confers ferroptosis resistance Regulation of metabolic flux through the transsulfuration pathway is well controlled at enzymatic reaction level. Two main enzymes, CBS, which catalyses the condensation of homocysteine to generate cystathionine, and CSE, which metabolises cystathionine into cysteine, are subject to regulatory control (Fig. 3a). To understand the mechanism underlying the upregulated transsulfuration pathway, we compared the expressions of CBS and CSE in 
a

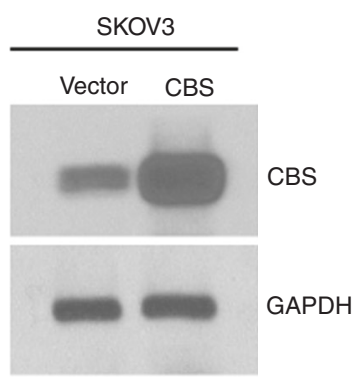

OVCA429


C11-BODIPY fluorescence (Mean: FL1-H)
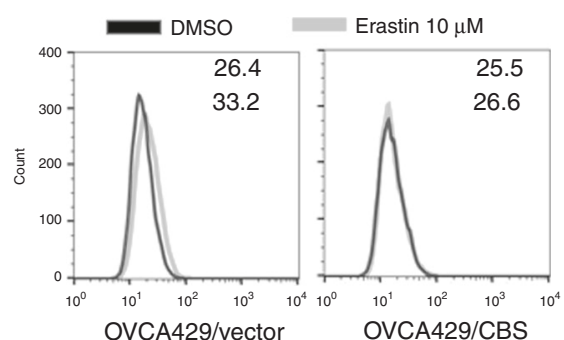

OVCA429/CBS
S-adenosyl homocysteine Homocysteine

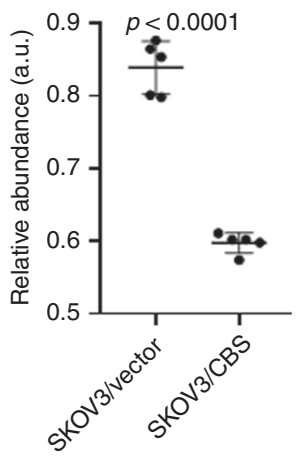

S-adenosyl homocysteine

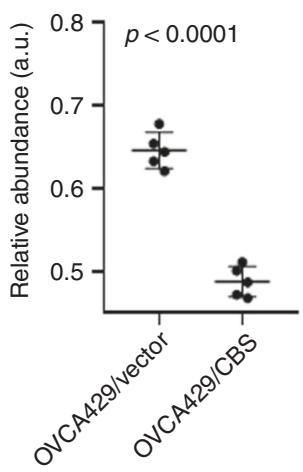

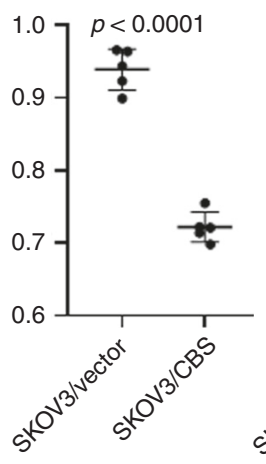

Homocysteine

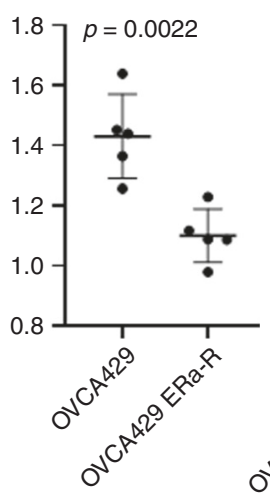

Cystathionine

Cysteine

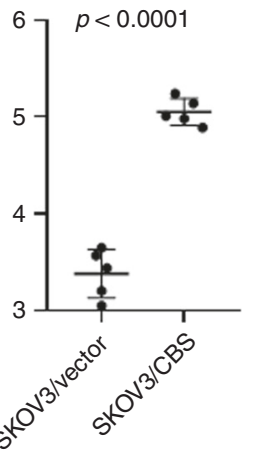

Cystathionine



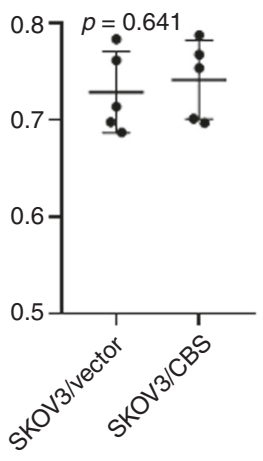

Cysteine
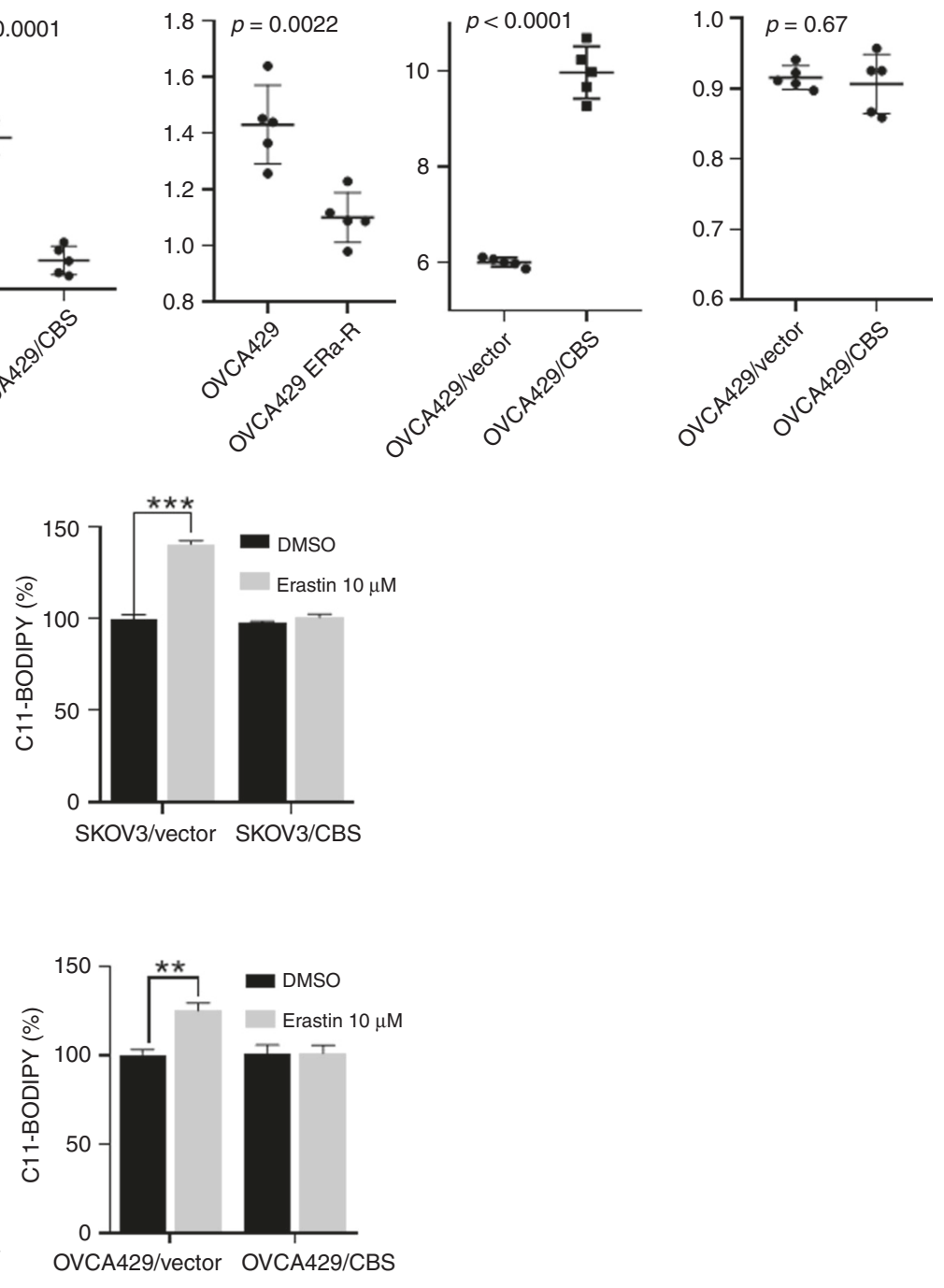

Fig. 4 CBS overexpression elevates the flux through the transsulfuration pathway and mitigates ferroptosis. a Cells were infected with lentivector or lenti-CBS for $48 \mathrm{~h}$. Left panel: CBS expression was analysed by western blotting. Right panel: cellular S-adenosyl homocysteine, homocysteine, cystathionine and cysteine were assessed. b, c Cells were infected with lentivector or lenti-CBS for 48 h, lipid ROS was analysed using C11-BODIPY probe (b), cellular GSH content was measured (c), ${ }^{* *} p<0.001,{ }^{* * *} p<0.0001$. d Cells were infected with lentivector or lentiCBS for $48 \mathrm{~h}$, followed by erastin $(10 \mu \mathrm{M})$ or SAS $(1 \mathrm{mM})$ treatment over $72 \mathrm{~h}$. Cell viability was examined, ${ }^{* *} p<0.001,{ }^{* * *} p<0.0001$.

parental cells and erastin-resistant cells. High levels of both protein and mRNA of CBS were found in erastin-resistant cells, while CSE protein did not show detectable difference between parental and erastin-resistant cells (Fig. 3b, c). Since the function of system $\mathrm{X}_{\mathrm{c}}{ }^{-}$is already blocked in erastin-resistant cells, as indicated by the inhibition of glutamate release to cell culture and the blockage of cystine uptake in Fig. $2 e, f$, we reasoned that CBS deficiency may cause cell death. Indeed, we observed that knocking down CBS by siRNA in erastin-resistant cells (Fig. 3d) triggered cell death (Fig. 3e). To clarify whether the CBS RNAi- 
C

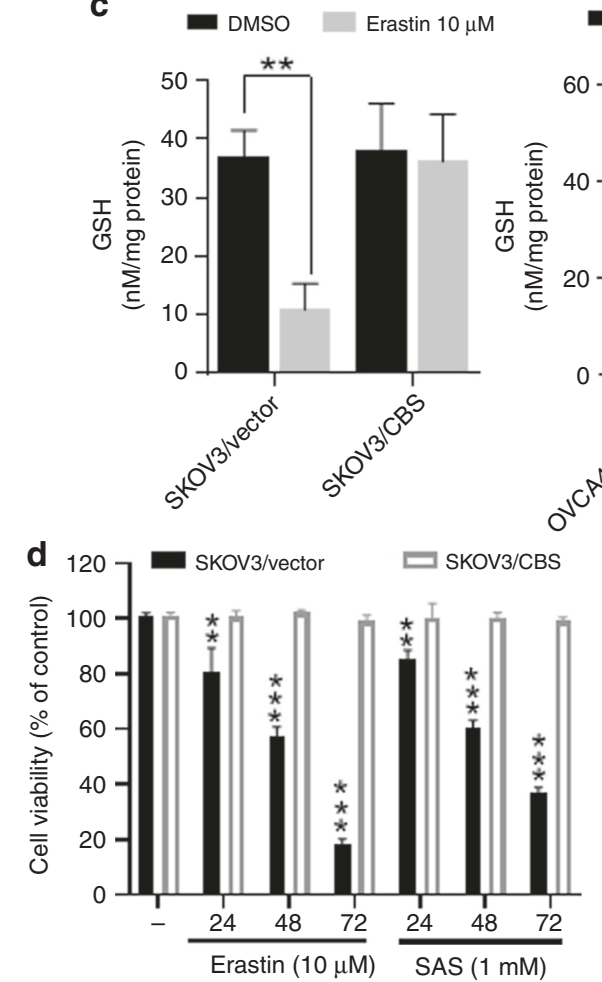

D DMSO Erastin $20 \mu \mathrm{M}$
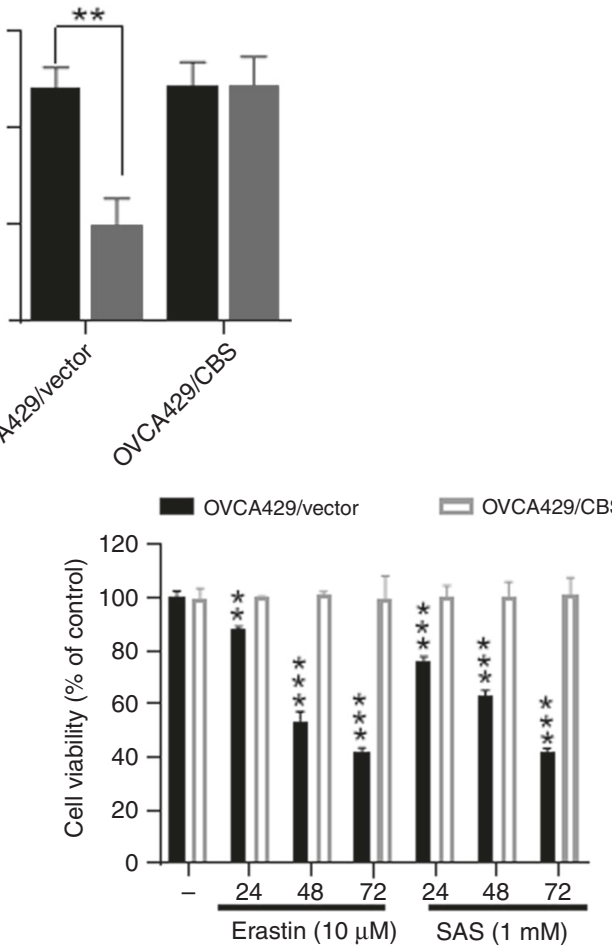

Fig. 4 Continued

induced cell death was mechanistically through ferroptosis pathway, we monitored the change in cellular GSH content, redox status and lipid peroxidation when CBS was depleted. As shown in Fig. 3f, GSH depletion was observed as early as $24 \mathrm{~h}$ after siRNA transfection, and the decrease of GSH was reversely correlated with the increase in cell death. Cellular ROS, as indicated by DCF fluorescence, and lipid peroxidation, as indicated by C11-BODIPY fluorescence (Fig. 3g) and MDA (Fig. 3h) production, were markedly enhanced by CBS knockdown. Furthermore, cellular lipid peroxidation was attenuated by the addition of ferroptosis inhibitors, Fer-1, as well as Lip-1 (Fig. 3i). CBS RNAiinduced cell death was also blocked by Fer-1 and Lip-1 (Fig. 3j), indicating that inhibition of CBS in the context of system $\mathrm{X}_{\mathrm{c}}{ }^{-}$ blockage caused ferroptosis. CBS is a primary enzyme to catalyze the production of $\mathrm{H}_{2} \mathrm{~S}_{1}^{33,34}$ which has been reported to contribute to cellular GSH content and scavenge lipid peroxidation. ${ }^{35,36}$ To assess whether CBS depletion by RNAi was accompanied with alteration of $\mathrm{H}_{2} \mathrm{~S}$ production, we quantified $\mathrm{H}_{2} \mathrm{~S}$ levels in erastinresistant cells receiving siCtrl or siCBS RNAs. Treatment of siCtrl cells with a CBS inhibitor, AOAA dramatically reduced the basal level of $\mathrm{H}_{2} \mathrm{~S}$, confirming the essential role of $\mathrm{CBS}$ in $\mathrm{H}_{2} \mathrm{~S}$ production. Basal $\mathrm{H}_{2} \mathrm{~S}$ was decreased $\sim 1.5-1.6$-fold in CBSknockdown cells, when compared with siCtrl cells (Fig. 3k). These data together suggested that erastin-induced ferroptosis resistance was dependent on the upregulation of CBS.

CBS overexpression elevates the flux through the transsulfuration pathway and mitigates ferroptosis

When CBS catalyses the initial and rate-limiting step of the transsulfuration pathway, ${ }^{37}$ we hypothesised that overexpression of CBS might increase the flux through the transsulfuration pathway, provide cells with alternative source of cysteine, thus rendering tolerance to cystine deprivation, as caused by erastin insult. To test this hypothesis, we overexpressed CBS in parental SKOV3 and OVCA429 cells (Fig. 4A). Metabolite profiles revealed the decrease in S-adenosyl homocysteine and homocysteine levels and the increase of cystathionine in CBS-overexpressed cells (Fig. 4a), indicating the enhanced flux of metabolites in the transsulfuration pathway. As expected, CBS overexpression dampened erastin-induced lipid peroxidation (Fig. 4b). GSH content was not much disturbed in CBS-overexpressing cells by erastin treatment (Fig. 4c). Strikingly, ferroptotic cell death induced by system $\mathrm{X}_{\mathrm{c}}{ }^{-}$inhibitor erastin, as well as SAS, was also rescued by CBS overexpression (Fig. 4d). Taken together, these data suggested that elevation of CBS mitigated ferroptosis by cystine deprivation via the enhanced transsulfuration pathway.

Activated NRF2 upregulates CBS

Accumulated evidences have positioned the antioxidant transcriptional factor NF-E2-related factor-2 (NRF2) to regulate ferroptosis through its target genes. ${ }^{38}$ To decipher how CBS was upregulated in erastin-resistant cells, we monitored NRF2, CBS and CSE abundance at different time points in response to erastin treatment. We observed a clear correlation with the induction of NRF2 and CBS as examined by immunoblotting (Fig. 5a). We noticed that erastinresistant cells exhibited higher basal level of NRF2 than that of parental cells (Fig. 5 lanes 5 and 1), indicating the constitutive activation of NRF2 in erastin-resistant cells. To determine the role of NRF2 in the activation of CBS, we silenced NRF2 by siRNA and analysed CBS protein and mRNA expression. Silencing NRF2 dramatically declined CBS and prevented the activation of CBS upon erastin treatment (Fig. 5b). We further observed that the diminished GSH content and the increased MDA in NRF2-deficient cells (Fig. 5c), indicate the perturbation of cell redox homoeostasis and the increase in lipid peroxidation. Consequently, NRF2 depletion abrogated cells resistance to erastin (Fig. 5d). NRF2 regulates antioxidant gene expression via activation of antioxidant response elements (AREs). ${ }^{39}$ The previous study suggested that mouse CBS is regulated by NRF2, likely through an ARE in the upstream region of the gene. ${ }^{40}$ We wondered whether human CBS promoter contains 
a

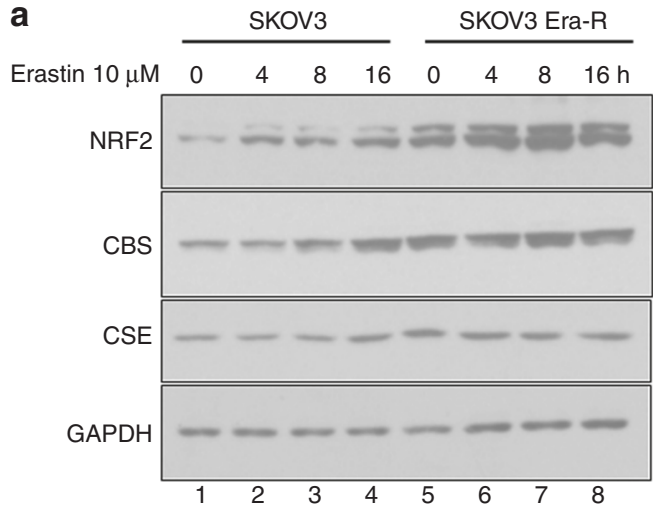

C $\quad$ DMSO
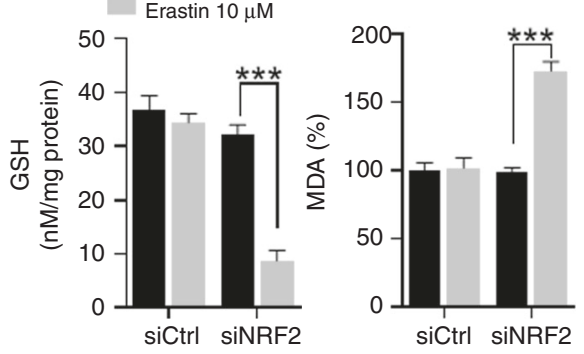

b

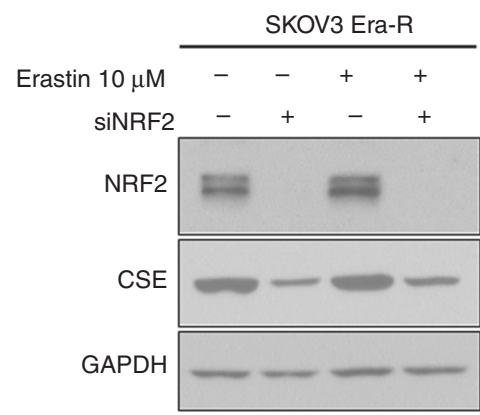

SKOV3 Era-R

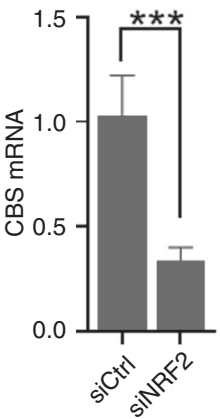

d

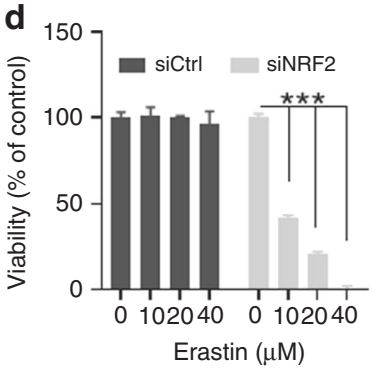

e

\begin{tabular}{|clll|}
\hline ARE consensus sequence & A C & & \\
Human CBS gene (NM_001178009) +67 GTGGGTCCCC & GTGAGNNNGCC & \\
GCGAGGCCGCC & ACCCCGGGGT & +97 \\
\hline
\end{tabular}

f
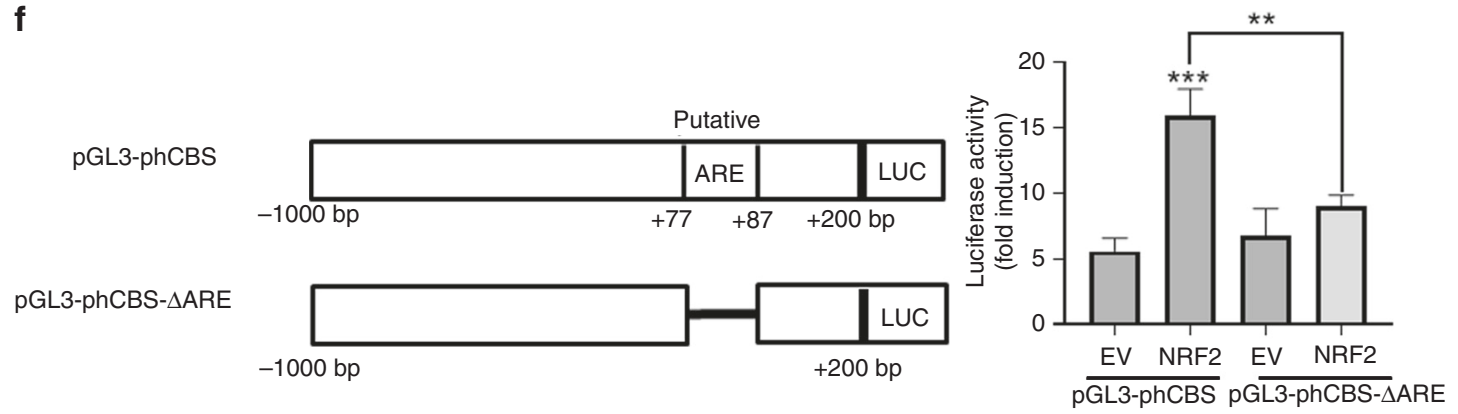

Fig. 5 Activated NRF2 upregulates CBS. a SKOV3 and SKOV3 Era-R cells were treated with erastin (10 $\mu$ M) for the indicated time; protein abundance was examined by western blotting. b SKOV3 Era-R cells were transfected with siCtrl or siNRF2 for $48 \mathrm{~h}$, then treated with or without erastin $(10 \mu \mathrm{M})$ for $24 \mathrm{~h}$. Left panel: protein analysis by western blotting; right panel: analysis of CBS mRNA level by qPCR, *** $p<0.0001$. c SKOV3 Era-R cells were treated as in (b); GSH content and MDA were analysed, ${ }^{* * *} p<0.0001$. d SKOV3 Era-R cells were transfected with siCtrl or siNRF2 for $48 \mathrm{~h}$, then treated with indicated doses of erastin for $24 \mathrm{~h}$. Cell viability was examined, ${ }^{* * *} p<0.0001$. e Potential ARE sequence in the human CBS promotor region. $\mathbf{f}$ Left panel: Diagram illustrating cloning of wild-type and deletion mutant of CBS promoter fragments into pGL3-basic luciferase vector. Right panel: The indicated reporter vector, together with pCDNA3 empty vector or pCDNA3-Myc3-NRF2 and pCH110 ( $\beta$-galactosidase-expressing plasmid) were cotransfected into SKOV3 Era-R cells. The activities of these reporter constructs were measured $24 \mathrm{~h}$ post transfection, ${ }^{* *} p<0.001,{ }^{* * *} p<0.0001$.

ARE. In silico examination of the human CBS genomic locus revealed that it contains one putative ARE sequence located in the proximal promoter region, approximately from +67 to $+97 \mathrm{bp}$, as illustrated in Fig. 5e. To determine whether this ARE is functional to mediate NRF2-dependent upregulation of CBS gene expression, we cloned CBS promoter fragment from -1000 to $+200 \mathrm{bp}$ and mutant fragment with deletion of +77 to $+87 \mathrm{bp}$ into a pGL3-basic luciferase reporter vector, which were designated as pGL3-phCBS and pGI3-phCBS- $\triangle A R E$, respectively. NRF2 overexpression in SKOV3 Era-R cells significantly increased the luciferase activity of reporter pGL3-phCBS plasmid, whereas depletion of the putative ARE in the promoter region of the CBS gene decreased the NRF2-induced luciferase reporter activity (Fig. 5f), indicating that the ARE is responsible for NRF2 regulation of CBS. Together, these results suggested that NRF2 was responsible for the upregulation of CBS in erastin-resistant cells.

\section{DISCUSSION}

Recent studies have shown the potential efficacy of ferroptosis inducer erastin in antitumour treatment ${ }^{22,41-43}$ and the synergism with chemotherapeutic agents in certain cancer cells. ${ }^{32,44}$ In an effort to investigate the effect of erastin in ferroptotic cell death in ovarian cancer cells, we found that erastin treatment could induce 


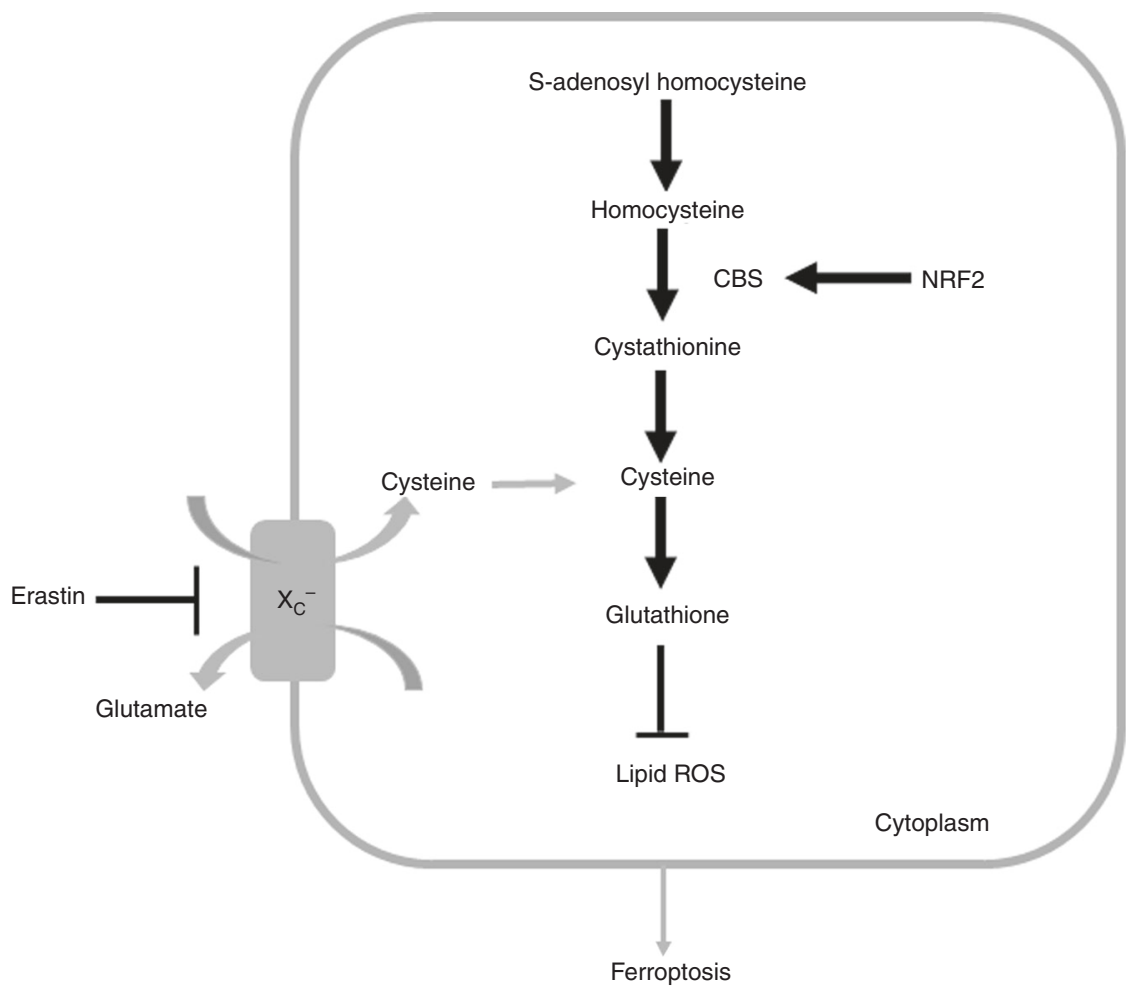

Fig. 6 Schematic model of activation of NRF2/CBS axis regulating ferroptosis resistance.

ferroptosis resistance. Upon erastin treatment, some cells exploited the transsulfuration pathway as a major source of cysteine, which counteracted the cysteine shortage due to system $\mathrm{X}_{\mathrm{c}}{ }^{-}$inhibition. We reported the regulatory role of the transsulfuration pathway in ferroptosis repertoire (Fig. 6).

The main treatment for ovarian cancer is debulking surgery followed by chemotherapy and/or radiation therapy. Despite an initially sensitive chemotherapy, most ovarian cancers relapse with chemoresistance, which is featured by genotypic alteration and metabolic changes. ${ }^{45-47}$ Thus, understanding the mechanisms during the progression to drug resistance may guide to develop effective therapeutic strategies to reduce ovarian cancer mortality. Ferroptosis, like apoptosis, is an exquisitely regulated cell death and is likely the adaptive process to remove malignant cells. ${ }^{48-50}$ We set out to search the potentially clinical use of ferroptosis inducers in cancer treatment. Notably, we observed that ferroptosis inducer erastin can also induce ferroptosis resistance in ovarian cancer cells. The finding is reminiscent of chemoresistance, which represents a major barrier for ovarian cancer therapy.

We further investigated the mechanism underlying ferroptosis resistance. In response to chronic erastin treatment, very few SKOV3 and OVCA429 cells developed the enhanced flux through the transsulfuration pathway and gradually adapted to cystine deprivation caused by system $\mathrm{X}_{\mathrm{c}}{ }^{-}$blockage. CBS catalyses the committing step in this pathway and is subject to multilevel regulation. ${ }^{51}$ Recent studies have shown the oncogenic role of CBS in colon and ovarian cancer models. ${ }^{52-55}$ We observed sustained upregulation of CBS in erastin-resistant cells, and further elucidated that upregulation of CBS was sufficient to render ferroptosis resistance. Knocking down CBS promoted cellular oxidative stress and lipid peroxidation, ultimately leading to ferroptosis. Overexpressing CBS enhanced the transsulfuration pathway and conferred ferroptosis resistance.

To examine how CBS was upregulated in erastin-resistant cells, we identified that NRF2 was constitutively activated and was positively correlated with CBS induction. We further identified a putative ARE in the human CBS promoter region and verified that ARE is responsible for NRF2-activated CBS promoter-driven luciferase activity. Furthermore, NRF2 inhibition caused CBS downregulation and sensitised cells to erastin result. We concluded that activation of NRF2/CBS accounts for ferroptosis resistance. We have not ruled out the possibility that other NRF2targeted genes might be involved in ferroptosis resistance; additional studies will be required to investigate the role of NRF2 in the inhibition of ferroptosis.

\section{ACKNOWLEDGEMENTS}

The authors thank Dr. Yanhong Yu for English assistance in the preparation of this paper.

\section{AUTHOR CONTRIBUTIONS}

N.L. designed experiments and prepared the paper. X.L. and C.H. performed research and analysed data.

\section{ADDITIONAL INFORMATION}

Competing interests: The authors declare no competing interests.

Ethics approval and consent to participate: Ethics approval was not required for this study. The SKOV3 cell line was purchased from ATCC (ATCC-HTB-77). OVCA429 cells were obtained from the Laboratory of Gynecologic Oncology at Brigham and Women's Hospital (Boston, Massachusetts, USA).

Funding: This work was supported by Science and Technology Planning Project of Guangdong Province of China (2014A020212188).

Consent to publish: Not applicable.

Data availability: All presented data are available from the corresponding author upon reasonable request. 
Note: This work is published under the standard license to publish agreement. After 12 months the work will become freely available and the license terms will switch to a Creative Commons Attribution 4.0 International (CC BY 4.0).

Publisher's note Springer Nature remains neutral with regard to jurisdictional claims in published maps and institutional affiliations.

\section{REFERENCES}

1. Bannai, S., Christensen, H. N., Vadgama, J. V., Ellory, J. C., Englesberg, E., Guidotti, G. G. et al. Amino acid transport systems. Nature 311, 308 (1984).

2. Bannai, S. \& Kitamura, E. Transport interaction of L-cystine and L-glutamate in human diploid fibroblasts in culture. J. Biol. Chem. 255, 2372-2376 (1980)

3. Banjac, A., Perisic, T., Sato, H., Seiler, A., Bannai, S., Weiss, N. et al. The cystine/ cysteine cycle: a redox cycle regulating susceptibility versus resistance to cell death. Oncogene 27, 1618-1628 (2008).

4. Conrad, M. \& Sato, H. The oxidative stress-inducible cystine/glutamate antiporter, system x (c) (-): cystine supplier and beyond. Amino Acids 42, 231-246 (2012).

5. Goji, T., Takahara, K., Negishi, M. \& Katoh, H. Cystine uptake through the cystine/ glutamate antiporter xCT triggers glioblastoma cell death under glucose deprivation. J Biol. Chem. 292, 19721-19732 (2017).

6. Sato, H., Tamba, M., Ishii, T. \& Bannai, S. Cloning and expression of a plasma membrane cystine/glutamate exchange transporter composed of two distinct proteins. J. Biol. Chem. 274, 11455-11458 (1999).

7. Sato, H., Tamba, M., Kuriyama-Matsumura, K., Okuno, S. \& Bannai, S. Molecular cloning and expression of human $\mathrm{xCT}$, the light chain of amino acid transport system xc. Antioxid. Redox. Signal. 2, 665-671 (2000).

8. Dixon, S. J., Lemberg, K. M., Lamprecht, M. R., Skouta, R., Zaitsev, E. M., Gleason, C. E. et al. Ferroptosis: an iron-dependent form of nonapoptotic cell death. Cell 149 1060-1072 (2012).

9. Xie, Y., Hou, W., Song, X., Yu, Y., Huang, J., Sun, X. et al. Ferroptosis: process and function. Cell Death Differ. 23, 369-379 (2016).

10. Dixon, S. J. \& Stockwell, B. R. The Hallmarks of Ferroptosis. Ann. Rev. Cancer Biol. 3 , 35-54 (2019)

11. Friedmann Angeli, J. P., Krysko, D. V. \& Conrad, M. Ferroptosis at the crossroads of cancer-acquired drug resistance and immune evasion. Nat. Rev. Cancer 19, 405-414 (2019).

12. Proneth, B. \& Conrad, M. Ferroptosis and necroinflammation, a yet poorly explored link. Cell Death Differ. 26, 14-24 (2019).

13. McBean, G. J. The transsulfuration pathway: a source of cysteine for glutathione in astrocytes. Amino Acids 42, 199-205 (2012).

14. Sbodio, J. I., Snyder, S. H. \& Paul, B. D. Regulators of the transsulfuration pathway. Br. J. Pharmacol. 176, 583-593 (2019).

15. Stipanuk, M. H., Dominy, J. E. Jr., Lee, J. I. \& Coloso, R. M. Mammalian cysteine metabolism: new insights into regulation of cysteine metabolism. J. Nutr. 136, 1652S-1659S (2006).

16. Kraus, J. P., Hasek, J., Kozich, V., Collard, R., Venezia, S., Janosikova, B. et al. Cystathionine gamma-lyase: Clinical, metabolic, genetic, and structural studies. Mol. Genet. Metab. 97, 250-259 (2009).

17. Bearden, S. E., Beard, R. S. Jr. \& Pfau, J. C. Extracellular transsulfuration generates hydrogen sulfide from homocysteine and protects endothelium from redox stress. Am. J. Physiol. Heart. Circ. Physiol. 299, H1568-1576 (2010).

18. Sugars, K. L. \& Rubinsztein, D. C. Transcriptional abnormalities in Huntington disease. Trends Genet. 19, 233-238 (2003).

19. Hensley, K. \& Denton, T. T. Alternative functions of the brain transsulfuration pathway represent an underappreciated aspect of brain redox biochemistry with significant potential for therapeutic engagement. Free Radic. Biol. Med. 78, 123-134 (2015)

20. Zhang, Y., Tan, H., Daniels, J. D., Zandkarimi, F., Liu, H., Brown, L. M. et al. Imidazole ketone erastin induces ferroptosis and slows tumor growth in a mouse lymphoma model. Cell Chem. Biol. 26, 623-633 (2019).

21. Skouta, R., Dixon, S. J., Wang, J., Dunn, D. E., Orman, M., Shimada, K. et al. Ferrostatins inhibit oxidative lipid damage and cell death in diverse disease models. J Am Chem Soc 136, 4551-4556 (2014).

22. Gout, P. W., Buckley, A. R., Simms, C. R. \& Bruchovsky, N. Sulfasalazine, a potent suppressor of lymphoma growth by inhibition of the $\mathrm{x}(\mathrm{c})$ - cystine transporter: a new action for an old drug. Leukemia 15, 1633-1640 (2001).

23. Tarangelo, A., Magtanong, L., Bieging-Rolett, K. T., Li, Y., Ye, J., Attardi, L. D. et al. p53 Suppresses metabolic stress-induced ferroptosis in cancer cells. Cell Rep 22, 569-575 (2018)

24. Dixon, S. J., Patel, D. N., Welsch, M., Skouta, R., Lee, E. D., Hayano, M. et al. Pharmacological inhibition of cystine-glutamate exchange induces endoplasmic reticulum stress and ferroptosis. Elife 3, e02523 (2014).
25. Wang, L., Cai, H., Hu, Y., Liu, F., Huang, S., Zhou, Y. et al. A pharmacological probe identifies cystathionine beta-synthase as a new negative regulator for ferroptosis. Cell Death Dis. 9, 1005 (2018).

26. Fusco, F., di Villa Bianca, R., Mitidieri, E., Cirino, G., Sorrentino, R. \& Mirone, V. Sildenafil effect on the human bladder involves the L-cysteine/hydrogen sulfide pathway: a novel mechanism of action of phosphodiesterase type 5 inhibitors. Eur. Urol. 62, 1174-1180 (2012).

27. d'Emmanuele di Villa Bianca, R., Mitidieri, E., Di Minno, M. N., Kirkby, N. S., Warner, T. D., Di Minno, G. et al. Hydrogen sulphide pathway contributes to the enhanced human platelet aggregation in hyperhomocysteinemia. Proc. Natl Acad. Sci. USA 110, 15812-15817 (2013).

28. Friedmann Angeli, J. P., Schneider, M., Proneth, B., Tyurina, Y. Y., Tyurin, V. A., Hammond, V. J. et al. Inactivation of the ferroptosis regulator Gpx4 triggers acute renal failure in mice. Nat. Cell Biol. 16, 1180-1191 (2014).

29. Slee, E. A., Zhu, H., Chow, S. C., MacFarlane, M., Nicholson, D. W. \& Cohen, G. M. Benzyloxycarbonyl-Val-Ala-Asp (OMe) fluoromethylketone (Z-VAD.FMK) inhibits apoptosis by blocking the processing of CPP32. Biochem. J. 315, 21-24 (1996).

30. Shimada, K., Skouta, R., Kaplan, A., Yang, W. S., Hayano, M., Dixon, S. J. et al. Global survey of cell death mechanisms reveals metabolic regulation of ferroptosis. Nat. Chem. Biol. 12, 497-503 (2016).

31. Marrocco, I., Altieri, F. \& Peluso, I. Measurement and clinical significance of biomarkers of oxidative stress in humans. Oxid. Med. Cell Longev. 2017, 6501046 (2017)

32. Sato, M., Kusumi, R., Hamashima, S., Kobayashi, S., Sasaki, S., Komiyama, Y. et al. The ferroptosis inducer erastin irreversibly inhibits system xc- and synergizes with cisplatin to increase cisplatin's cytotoxicity in cancer cells. Sci. Rep. 8, 968 (2018).

33. Singh, S., Padovani, D., Leslie, R. A., Chiku, T. \& Banerjee, R. Relative contributions of cystathionine beta-synthase and gamma-cystathionase to $\mathrm{H} 2 \mathrm{~S}$ biogenesis via alternative trans-sulfuration reactions. J. Biol. Chem. 284, 22457-22466 (2009).

34. Majtan, T., Krijt, J., Sokolova, J., Krizkova, M., Ralat, M. A., Kent, J. et al. Biogenesis of hydrogen sulfide and thioethers by cystathionine beta-synthase. Antioxid. Redox Signal. 28, 311-323 (2018).

35. Kimura, Y., Goto, Y. \& Kimura, H. Hydrogen sulfide increases glutathione production and suppresses oxidative stress in mitochondria. Antioxid. Redox Signal. 12, 1-13 (2010).

36. Schreier, S. M., Muellner, M. K., Steinkellner, H., Hermann, M., Esterbauer, H., Exner, $M$. et al. Hydrogen sulfide scavenges the cytotoxic lipid oxidation product 4-HNE. Neurotox. Res. 17, 249-256 (2010).

37. Beard, R. S. Jr. \& Bearden, S. E. Vascular complications of cystathionine betasynthase deficiency: future directions for homocysteine-to-hydrogen sulfide research. Am. J. Physiol. Heart Circ. Physiol. 300, H13-26 (2011).

38. Abdalkader, M., Lampinen, R., Kanninen, K. M., Malm, T. M. \& Liddell, J. R. Targeting Nrf2 to Suppress ferroptosis and mitochondrial dysfunction in neurodegeneration. Front. Neurosci. 12, 466 (2018)

39. Kuosmanen, S. M., Viitala, S., Laitinen, T., Perakyla, M., Polonen, P., Kansanen, E. et al. The effects of sequence variation on genome-wide NRF2 binding-new target genes and regulatory SNPs. Nucleic Acids Res. 44, 1760-1775 (2016).

40. Hourihan, J. M., Kenna, J. G. \& Hayes, J. D. The gasotransmitter hydrogen sulfide induces nrf2-target genes by inactivating the keap1 ubiquitin ligase substrate adaptor through formation of a disulfide bond between cys-226 and cys- 613 . Antioxid. Redox Signal. 19, 465-481 (2013).

41. Yu, H., Guo, P., Xie, X., Wang, Y. \& Chen, G. Ferroptosis, a new form of cell death and its relationships with tumourous diseases. J. Cell. Mol. Med. 21, 648-657 (2017)

42. Liu, D. S., Duong, C. P., Haupt, S., Montgomery, K. G., House, C. M., Azar, W. J. et al. Inhibiting the system $\times C(-) / g l$ lutathione axis selectively targets cancers with mutant-p53 accumulation. Nat. Commun. 8, 14844 (2017).

43. Yu, Y., Xie, Y., Cao, L., Yang, L., Yang, M., Lotze, M. T. et al. The ferroptosis inducer erastin enhances sensitivity of acute myeloid leukemia cells to chemotherapeutic agents. Mol. Cell Oncol. 2, e1054549 (2015).

44. Chen, L., Li, X., Liu, L., Yu, B., Xue, Y. \& Liu, Y. Erastin sensitizes glioblastoma cells to temozolomide by restraining $\mathrm{xCT}$ and cystathionine-gamma-lyase function. Oncol. Rep. 33, 1465-1474 (2015).

45. Schorge, J. O., McCann, C. \& Del Carmen, M. G. Surgical debulking of ovarian cancer: what difference does it make?. Rev. Obstet. Gynecol. 3, 111-117 (2010).

46. Li, S. S., Ma, J. \& Wong, A. S. T. Chemoresistance in ovarian cancer: exploiting cancer stem cell metabolism. J. Gynecol. Oncol. 29, e32 (2018).

47. Levine, A. J. \& Puzio-Kuter, A. M. The control of the metabolic switch in cancers by oncogenes and tumor suppressor genes. Science 330, 1340-1344 (2010).

48. Brasseur, K., Gevry, N. \& Asselin, E. Chemoresistance and targeted therapies in ovarian and endometrial cancers. Oncotarget 8, 4008-4042 (2017).

49. Lewerenz, J., Ates, G., Methner, A., Conrad, M. \& Maher, P. Oxytosis/Ferroptosis(Re-) emerging roles for oxidative stress-dependent non-apoptotic cell death in diseases of the central nervous system. Front. Neurosci. 12, 214 (2018). 
50. Mou, Y., Wang, J., Wu, J., He, D., Zhang, C., Duan, C. et al. Ferroptosis, a new form of cell death: opportunities and challenges in cancer. J. Hematol. Oncol. 12, 34 (2019).

51. Prudova, A., Bauman, Z., Braun, A., Vitvitsky, V., Lu, S. C. \& Banerjee, R. Sadenosylmethionine stabilizes cystathionine beta-synthase and modulates redox capacity. Proc. Natl Acad. Sci. USA 103, 6489-6494 (2006).

52. Phillips, C. M., Zatarain, J. R., Nicholls, M. E., Porter, C., Widen, S. G., Thanki, K. et al. Upregulation of cystathionine-beta-synthase in colonic epithelia reprograms metabolism and promotes carcinogenesis. Cancer Res. 77, 5741-5754 (2017).
53. Jin, S., Chen, Z., Ding, X., Zhao, X., Jiang, X., Tong, Y. et al. Cystathionine-betasynthase inhibition for colon cancer: Enhancement of the efficacy of aminooxyacetic acid via the prodrug approach. Mol Med. 22, 54-63 (2016).

54. Bhattacharyya, S., Saha, S., Giri, K., Lanza, I. R., Nair, K. S., Jennings, N. B. et al. Cystathionine beta-synthase (CBS) contributes to advanced ovarian cancer progression and drug resistance. PLoS One 8, e79167 (2013).

55. Chakraborty, P. K., Xiong, X., Mustafi, S. B., Saha, S., Dhanasekaran, D., Mandal, N. A. et al. Role of cystathionine beta synthase in lipid metabolism in ovarian cancer. Oncotarget 6, 37367-37384 (2015). 